\title{
Local CSI Based Selection Beamforming for Amplify-and-Forward MIMO Relay Networks
}

\author{
Batu Krishna Chalise, Senior Member, IEEE, Luc Vandendorpe, Fellow, IEEE, \\ Yimin D. Zhang, Senior Member, IEEE, and Moeness G. Amin, Fellow, IEEE
}

\begin{abstract}
We investigate selection beamforming for a cooperative network that consists of a source, a destination, and two amplify-and-forward (AF) relays, which are all equipped with multiple antennas. The transmit and receive beamforming techniques are respectively applied at the source and destination, and the linear processing operator of the selected AF multiple-input multiple-output (MIMO) relay is optimized. We consider that the source has the instantaneous channel state information (CSI) of the channels from the source to relays but no information regarding the CSI of the channels from the relays to the destination. Partial relay selection (PRS) is employed, i.e., the source routes its information signal to the relay which offers better first-hop signal-to-noise ratio (SNR). Considering a Rayleigh fading environment, we derive an exact closed-form expression for the outage probability at the destination. The validity of the outage probability expression has been confirmed with numerical simulations. We also present the asymptotic analysis of the PRS scheme for the considered multiantenna system and derive the expressions of diversity gain. It is shown that, the overall diversity gain is $\min \left(\min \left(n_{\mathrm{r}, 1} n_{\mathrm{d}}, n_{\mathrm{r}, 2} n_{\mathrm{d}}\right), n_{\mathrm{r}, 1} n_{\mathrm{s}}+n_{\mathrm{r}, 2} n_{\mathrm{s}}\right)$ where $n_{\mathrm{s}}, n_{\mathrm{d}}$, and $n_{\mathrm{r}, q}$ are, respectively, the numbers of antennas at the source, destination and the $q$ th relay. For the condition $C: \min \left(n_{\mathrm{r}, 1} n_{\mathrm{d}}, n_{\mathrm{r}, 2} n_{\mathrm{d}}\right) \geq n_{\mathrm{r}, 1} n_{\mathrm{s}}+n_{\mathrm{r}, 2} n_{\mathrm{s}}$ that can be easily met with practically realizable antenna configurations, it is shown that PRS provides the same diversity order as opportunistic relaying $(\mathrm{OR})$.
\end{abstract}

Index Terms-Amplify-and-forward MIMO relay, beamforming, opportunistic relaying, outage probability, partial relay selection.

\section{INTRODUCTION}

$\mathbf{U}$ SER COOPERATION DIVERSITY, or cooperative diversity, [1], [2] has emerged as a promising technique to improve spatial diversity gain in wireless networks. The key phenomenon in cooperative communications is that a source takes the help of user terminals that are in its coverage area to relay its signal to the destination. Since the destination sees the replicas (most likely independent copies) of the source signal,

Manuscript received April 14, 2011; revised September 20, 2011 and December 22, 2011; accepted January 03, 2012. Date of publication January 16, 2012; date of current version April 13, 2012. The associate editor coordinating the review of this manuscript and approving it for publication was Dr. Xavier Mestre.

B. K. Chalise, Y. D. Zhang, and M. G. Amin are with the Center for Advanced Communications, Villanova University, Villanova, PA 19085 USA (e-mail: batu.chalise@villanova.edu; yimin.zhang@villanova.edu; moeness.amin@villanova.edu).

L. Vandendorpe is with the ICTEAM Institute, Universitè catholique de Louvain, 1348-Louvain La Neuve, Belgium (e-mail: luc.vandendorpe@uclouvain. be).

Color versions of one or more of the figures in this paper are available online at http://ieeexplore.ieee.org.

Digital Object Identifier 10.1109/TSP.2012.2184535 the diversity gain will be enhanced. Various relaying protocols for cooperative communications such as amplify-and-forward (AF), decode-and-forward (DF) [2], coded-cooperation [3], and compress-and-forward [4] have been presented. Among them, the AF scheme is attractive due to its simplicity, since the relay simply forwards the signal and does not need to decode it. However, the application of more than one relay increases feedback requirements and the overall implementation cost. Moreover, in order to avoid mutual interference as well as to achieve full diversity and multiplexing gains, the beamformers or space-time codes applied over the set of relays need to be jointly optimized. Such optimization also requires strict time and phase synchronization among the relay nodes. Towards this end, relay selection has been recognized as an effective way to reduce signaling overhead and complexity but keep intact the benefits of multiuser relay cooperation. The basic idea in relay selection is to select the best relay from a number of available relays, according to some well defined criteria. The significant results in the area of relay selection appeared in [5], where relay selection schemes are based on instantaneous signal-to-noise ratios (SNRs) of both source-relay and relay-destination links. In the literature [6]-[10], several other relay selection methods [also known as opportunistic relaying (OR)] have been proposed, which mainly rely on instantaneous SNR of two-hop links from the source to the destination. ${ }^{1}$ Another common aspect of all of these works is that they have been limited to cooperative systems where all nodes are equipped with a single antenna.

It has been shown that (see [11] and the references therein) the performance of cooperative relay systems can be further enhanced by accommodating multiple antennas at each node and thus forming multiple-input multiple-output (MIMO) diversity systems. The bounds on the diversity-multiplexing tradeoff for cooperative MIMO relay systems have been analyzed in [12] for different relay protocols. In [13], assuming Rayleigh fading, the outage probability of the AF protocol is derived in a multihop MIMO relay network that employs transmit antenna selection (TAS) and maximum ratio combining (MRC). However, there have been very few works that extend the relay selection protocols of [5], [6] to a multiantenna relay network. Recently, the authors of [14] propose joint relay-and-antenna selection schemes using TAS and extend the selection schemes of [5] and [6] to a network with multiple multiantenna DF relays. The proposed selection schemes of [14], however, require the destination to have the knowledge of instantaneous SNRs of all links from the source to relays and from the relays to the destination.

\footnotetext{
${ }^{1}$ Note that the two-hop SNR is composed of the SNRs of links from source to the relay and from the relay to the destination.
} 
It is worthwhile to note that relay systems having perfect global channel state information (CSI) and all multiantenna nodes (source, destination and relays) have been investigated in [15] and [16], from an optimization perspective rather than from a performance analysis perspective. In [15], it is shown that the joint optimization of source and relay precoders is a nonconvex optimization problem in general, where suboptimal solutions are obtained using an iterative approach. In [16], the authors preselect a limited number of relays that have better two-hop channel quality and employ semi-orthogonality among either spatial eigenmodes or antenna pairs of the preselected relays for jointly optimizing the source and relay precoder matrices.

Due to various reasons, it is generally difficult to obtain perfect global CSI. Collecting the global CSI at a central node demands significant amount of traffic, particularly in a fast time-varying environment and is undesirable for low-cost systems where bandwidth restrictions can be severe (e.g., ad hoc and sensor networks) [17]. An alternative approach that does not require global CSI at the central node or each relay node was proposed in [18]. This scheme, known as partial relay selection (PRS), selects the best relay according to the CSI of only the channels from the source to relays and thus reduces signaling overhead required for cooperation. In [18]-[21], the performance of PRS scheme is analyzed for CSI-assisted and fixed-gain AF relaying systems, respectively. However, all of these works [18]-[21] assume that all nodes are equipped with a single antenna. Recently, [22] studies diversity performance of PRS method for a CSI-assisted AF relaying system that consists of a single-antenna source, multiple single-antenna relays, and a multiantenna destination.

In this paper, we investigate selection beamforming technique for a relay network, in which the source, destination, and two AF relay nodes are all equipped with multiple antennas. Linear processing schemes (i.e., beamforming and MIMO relay precoding) are applied to the appropriate nodes, and the source and the relays are considered to transmit with some power constraints. Instead of assuming global CSI at one or more nodes, we consider practical scenario of interest, in which the nodes have instantaneous CSI of only local channels. In particular, the source knows its channels to the relays, the destination has the CSI of its channels with the relays, and each relay knows its channels with the source and destination. Based on this local CSI, the PRS scheme is employed, i.e., the relay that gives better SNR in the first-hop link is selected. In this case, the optimal source and destination beamformers, and the relay precoders have the same simple closed-form solutions as in the case of a two-hop relay system with no direct link from the source to the destination [23]. Based on these solutions, the exact outage probability at the destination node is derived in closed-form for a Rayleigh fading environment. We also derive an asymptotic expression of the outage probability for determining the diversity order of the system.

To the best of our knowledge, performance analysis for PRS with beamforming at all multiantenna nodes is a new problem that has not been investigated and solved before. The problem is interesting because the exact diversity order of the PRS scheme for the considered system model is not previously known. Moreover, the problem is challenging since the state-of-the-art technology [5]-[10], [13], [14], [18]-[22] used for the performance analysis of relay selection schemes are either not applicable or not easily extendable for our considered setup of multiantenna nodes with beamforming. Note that the outage probability at the destination can be readily expressed as the product of the outage probabilities of individual source-relay-destination (two-hop) links when OR is used (cf. Remark 4, Section IV). Consequently, in such a case the final closed-form expression easily follows from the outage probability expressions of the two-hop channels. Similarly, with some simple steps, it can be shown that OR achieves full diversity (cf. Remark 4, Section IV). However, unlike in the case of OR, neither the exact outage probability expression nor the exact diversity gain of the PRS scheme is known. These facts have motivated us to study PRS instead of OR. In particular, our objective is to answer the following two key questions:

1) Knowing that the performance of PRS is inferior to that of $\mathrm{OR}$, how does the former's performance degrade in terms of the outage probability?

2) Can full diversity be ever achieved in PRS?

This paper provides analytical results to answer both questions. With these discussions on the motivations, we are now ready to summarize the main contributions of this paper as follows.

- We derive an exact closed-form expression for the outage probability at the destination.

- The asymptotic analysis of the outage probability is presented to derive the diversity order.

- It is shown that the diversity order of PRS for the considered setup is given by

$$
\min \left(\min \left(n_{\mathrm{r}, 1} n_{\mathrm{d}}, n_{\mathrm{r}, 2} n_{\mathrm{d}}\right), n_{\mathrm{r}, 1} n_{\mathrm{s}}+n_{\mathrm{r}, 2} n_{\mathrm{s}}\right)
$$

where $n_{\mathrm{s}}$ and $n_{\mathrm{d}}$ are the numbers of antennas at the source and destination, respectively, and $n_{\mathrm{r}, q}$ is the number of antennas of the $q$ th relay with $q=1,2$.

- For the condition $C: \min \left(n_{\mathrm{r}, 1} n_{\mathrm{d}}, n_{\mathrm{r}, 2} n_{\mathrm{d}}\right) \geq n_{\mathrm{r}, 1} n_{\mathrm{s}}+$ $n_{\mathrm{r}, 2} n_{\mathrm{s}}$ that can be easily met with practically realizable antenna configurations, it is shown that PRS provides the same diversity order as OR.

The main challenge in the performance analysis for the proposed scheme originates from the statistical dependence of the SNR at the destination and the decision criterion employed at the source for routing. ${ }^{2}$ In particular, the outage probability in the proposed scenario include sum of the joint probabilities, each consists of statistically dependent random events with two or more continuous random variables. As a result, the derivation of the exact outage probability requires solving multidimensional integrals that need rigorous analysis. Moreover, the asymptotic analysis of the proposed scheme also needs to deal with the joint probabilities of statistically dependent random variables. According to our best source of knowledge, such a problem has not been solved in the literature.

The rest of the paper is organized as follows. The system model is described in Section II. The outage probability expressions are derived in Section III, and the corresponding asymp-

\footnotetext{
${ }^{2}$ Throughout this paper, the term routing is regularly used for relay selection since the decision to select the best relay is made at the source and based on the SNRs of only the channels from the source to the relays.
} 


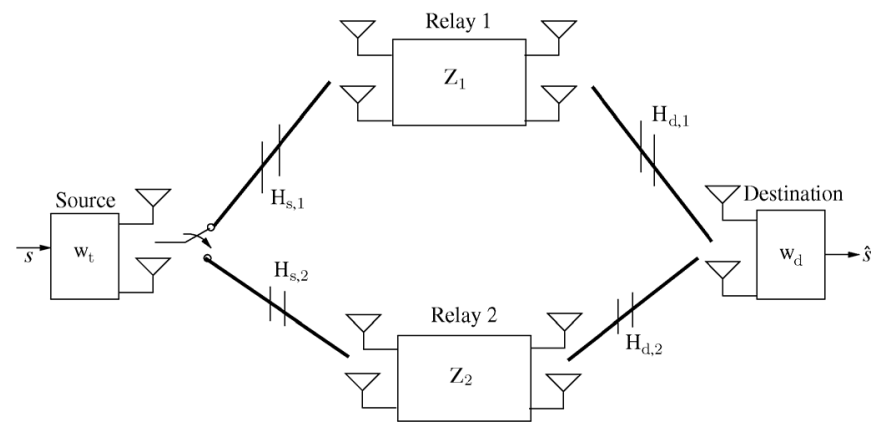

Fig. 1. Physical layer routing scheme for a multiantenna cooperative system.

totic results are derived in Section IV. In Section V, analytical and simulation results are provided. Section VI concludes the paper.

Notations: Upper (lower) boldface letters will be used for matrices (vectors); $(\cdot)^{H}, f_{\alpha}(x), \operatorname{Pr}\{\cdot\}$, and $\mathrm{E}\{\}$ denote Hermitian transpose, probability density function (PDF) of a continuous random variable $\alpha$, the probability operator and the mathematical expectation, respectively. $\operatorname{Pr}\{\mathcal{A}, \mathcal{B}\}$ denotes the joint probability of the random events $\mathcal{A}$ and $\mathcal{B}$, and $\mathcal{C}^{M \times M}$ stands for the space of $M \times M$ matrices with complex entries.

\section{System MODEL}

We study a cooperative network where signal transmission from the multiantenna source to the multiantenna destination is supported by two multiantenna relay terminals. The block diagram for this system is shown in Fig. 1. It is assumed that all nodes share the same frequency band. The source and destination have $n_{\mathrm{s}}$ and $n_{\mathrm{d}}$ antennas, respectively, whereas the $q$ th relay has $n_{\mathrm{r}, q}$ antennas where $q=1,2$. The MIMO channels between the source and relays are represented by $\left\{\mathbf{H}_{\mathrm{s}, q}\right\}_{q=1}^{2} \in \mathcal{C}^{n_{\mathrm{r}, q} \times n_{\mathrm{s}}}$, whereas the channels between the relays and destination are denoted by $\left\{\mathbf{H}_{\mathrm{d}, q}\right\}_{q=1}^{2} \in \mathcal{C}^{n_{\mathrm{d}} \times n_{\mathrm{r}, q}}$. The path attenuation factors associated with the channels $\left\{\mathbf{H}_{\mathrm{s}, q}\right\}_{q=1}^{2}$ and $\left\{\mathbf{H}_{\mathrm{d}, q}\right\}_{q=1}^{2}$ are denoted by $\left\{\sqrt{c_{\mathrm{s}, q}}\right\}_{q=1}^{2}$ and $\left\{\sqrt{c_{\mathrm{d}, q}}\right\}_{q=1}^{2}$, respectively. We assume a Rayleigh flat-fading environment where the channels are also considered to be spatially uncorrelated. Thus, the entries of $\left\{\mathbf{H}_{\mathrm{s}, q}\right\}_{q=1}^{2}$ and $\left\{\mathbf{H}_{\mathrm{d}, q}\right\}_{q=1}^{2}$ are assumed to be independent, zero-mean circularly symmetric complex Gaussian (ZMCSCG) random variables with unit variance. We also assume that shadowing effect is negligible in all the source-relay and relay-destination links, whereas the direct link between the source and destination is neglected due to higher path attenuation and possibly line-of-sight obstruction. We define the singular value decompositions (SVDs) of $\mathbf{H}_{\mathrm{s}, q}$ and $\mathbf{H}_{\mathrm{d}, q}$ as $\mathbf{H}_{\mathrm{s}, q} \triangleq \sum_{r=1}^{R_{q, 1}} \sigma_{\mathrm{s}, q}^{r} \mathbf{u}_{\mathrm{s}, q}^{r}\left(\mathbf{v}_{\mathrm{s}, q}^{r}\right)^{H}$ and $\mathbf{H}_{\mathrm{d}, q} \triangleq \sum_{r=1}^{R_{q, 2}} \sigma_{\mathrm{d}, q}^{r} \mathbf{u}_{\mathrm{d}, q}^{r}\left(\mathbf{v}_{\mathrm{d}, q}^{r}\right)^{H}$, respectively, for all $q$. Here, $\sigma_{\mathrm{s}, q}^{r}$ and $\sigma_{\mathrm{d}, q}^{r}$ are the singular values in the descending order (i.e., $\sigma_{\mathrm{s}, q}^{1} \geq \sigma_{\mathrm{s}, q}^{2} \cdots \geq \sigma_{\mathrm{s}, q}^{R_{q, 1}}$ and $\sigma_{\mathrm{d}, q}^{1} \geq \sigma_{\mathrm{d}, q}^{2} \cdots \geq \sigma_{\mathrm{d}, q}^{R_{q, 2}}$ ), $R_{q, 1}$ and $R_{q, 2}$ are the ranks of $\mathbf{H}_{\mathrm{s}, q}$ and $\mathbf{H}_{\mathrm{d}, q}$, respectively, and $\mathbf{u}_{\mathrm{s}, q}^{r} / \mathbf{v}_{\mathrm{s}, q}^{r}$ and $\mathbf{u}_{\mathrm{d}, q}^{r} / \mathbf{v}_{\mathrm{d}, q}^{r}$ are their respective left/right singular vectors. After these descriptions on system model, in the following subsection, we describe how the nodes acquire CSI required for relay selection and beamforming.

\section{A. CSI Acquisition and Transmission Protocol}

CSI acquisition: Note that, in order to implement relay selection and beamforming, local CSI is required at each node, i.e., the source needs to obtain the CSI of all source-relay $\left(\mathcal{S}-\mathcal{R}_{q}\right)$ channels, the $q$ th relay needs to obtain the CSI of the $\mathcal{S}-\mathcal{R}_{q}$ and relay-destination $\left(\mathcal{R}_{q}-\mathcal{D}\right)$ channels, and the destination needs to have the CSI of all $\mathcal{R}_{q}-\mathcal{D}$ links. For this purpose, all nodes transmit their training signals sequentially so that their respective neighboring nodes can estimate the local CSI. Because we assume that all the nodes share the same frequency band, CSI estimated at each transmit node (e.g., source, relays) can be considered as the transmit CSI due to channel reciprocity. The estimated CSI at all nodes are assumed to have negligible uncertainties. This assumption is valid in a slowly time-varying channel environment if the training signals are properly designed. After the training phase completes, it is easy to see that the source has necessary CSI to identify the relay that provides better first-hop SNR. As will be described later (cf. Data Transmission Protocol below), at the end of the training phase, the data transmission phase starts in which the nodes perform beamforming.

It is worthwhile to mention that the training-based CSI acquisition scheme employed in our system does not incur major penalty in terms of spectral efficiency provided that the channels are slowly time-varying, particularly when the SNR is sufficiently high enough [24]. Moreover, it is reported in recent experimental results [25] that there exists complexity and performance tradeoff between training-based (also called the reciprocity based method) and feedback-based CSI acquisition methods, and thus, the performance of the former method is comparable to the latter method.

Data Transmission Protocol: Each symbol transmission is considered over two time slots. At the beginning of each transmission (first time slot), based on the local CSI obtained during the training phase, the source determines its route for signal transmission. If the source finds that the $\mathcal{S}-\mathcal{R}_{1}$ channel is stronger than the $\mathcal{S}-\mathcal{R}_{2}$ channel, the source broadcasts its symbol by matching the beamformer $\mathbf{w}_{\mathrm{t}} \in \mathcal{C}^{n_{\mathrm{s}} \times 1}$ to the $\mathcal{S}-\mathcal{R}_{1}$ link. Otherwise, the source matches its beamformer to the $\mathcal{S}-\mathcal{R}_{2}$ channel and broadcasts its symbol. In either case, the source appends a flag-bit with the information symbol in order to notify the selected relay its selection for transmitting the source signal to the destination. The selected relay processes its received signal with the optimized relay beamforming matrix and transmits the resulting signal to the destination in the second time slot. In this way, the destination receives signals from one of the two relays, depending on the route selection carried out by the source. The following remarks are in order.

Remark 1: With the aforementioned framework of CSI acquisition, each node determines its beamformer locally after the training phase. Thus, there is no exchange of additional feedback information between the neighboring nodes during the data transmission phase, except the flag-bit that the source adds with its data.

Remark 2: The aforementioned data transmission protocol requires two time slots due to the assumption that the relays operate in a half-duplex mode. However, if the relays can support full-duplex transmission, e.g., if the cross interference between the retransmit and the receive signals can be suppressed using spatial nulling method [26], [27] or if the relay uses separate 
sets of directional antennas for transmission and reception [28], the transmission protocol can consist of only one time slot. It is important to emphasize that the statistical analysis of the considered protocol remains the same whether the relay is operating in full-duplex or half-duplex mode.

Let $q_{\mathrm{o}}$ be the index of the selected relay, where $q_{\mathrm{o}} \in\{1,2\}$. The signal received by this relay can be given by

$$
\mathbf{y}_{\mathrm{r}, q_{\mathrm{o}}}=\sqrt{c_{\mathrm{s}, q_{\mathrm{o}}}} \mathbf{H}_{\mathrm{s}, q_{\mathrm{o}}} \mathbf{w}_{\mathrm{t}} s+\mathbf{n}_{\mathrm{e}, q_{\mathrm{o}}} \in \mathcal{C}^{n_{\mathrm{r}, q_{\mathrm{o}}} \times 1}
$$

where $s$ is the random source signal having zero-mean and unitvariance (i.e., $\mathrm{E}\{s\}=0$ and $\mathrm{E}\left\{|s|^{2}\right\}=1$ ), $\mathbf{w}_{\mathrm{t}} \in \mathcal{C}^{n_{\mathrm{s}} \times 1}$ is the source beamformer and $\sqrt{c_{\mathrm{s}, q_{\mathrm{o}}}}$ is the distance-dependent path loss for the channel between the selected relay and the source. Moreover, in (1), $\mathbf{n}_{\mathrm{e}, q_{\mathrm{o}}}$ is the additive white Gaussian noise (AWGN) vector whose entries are assumed to be zeromean with the variance $\sigma_{n_{\mathrm{r}}}^{2}$, i.e., $\mathbf{n}_{\mathrm{e}, q_{\circ}} \sim \mathcal{N}_{C}\left(\mathbf{0}, \sigma_{n_{\mathrm{r}}}^{2} \mathbf{I}_{n_{\mathrm{r}, q_{\mathrm{o}}}}\right){ }^{3}$ The selected relay processes the received signal (1) with the

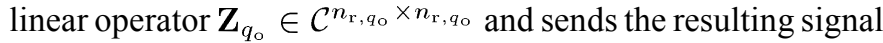
to the destination in the second time slot. Thus, the signal received by the destination is given by

$$
\mathbf{y}_{\mathrm{d}, q_{\mathrm{o}}}=\sqrt{c_{\mathrm{d}, q_{\mathrm{o}}}} \mathbf{H}_{\mathrm{d}, q_{\mathrm{o}}} \mathbf{Z}_{q_{\mathrm{o}}}\left(\sqrt{c_{\mathrm{s}, q_{\mathrm{o}}}} \mathbf{H}_{\mathrm{s}, q_{\mathrm{o}}} \mathbf{w}_{\mathrm{t}} s+\mathbf{n}_{\mathrm{R}, q_{\mathrm{o}}}\right)+\mathbf{n}_{\mathrm{D}, q_{\mathrm{o}}}
$$

where $\mathbf{n}_{\mathrm{D}, q_{\mathrm{o}}} \sim \mathcal{N}_{C}\left(\mathbf{0}, \sigma_{n_{\mathrm{d}}}^{2} \mathbf{I}_{n_{\mathrm{d}}}\right)$ represents the noise vector at the destination having ZMCSCG entries of variances $\sigma_{n_{\mathrm{d}}}^{2}$, and $\sqrt{c_{\mathrm{d}, q_{\mathrm{o}}}}$ is the distance-dependent path loss for the channel between the selected relay and the destination. The destination uses receive beamformer $\mathbf{w}_{\mathrm{d}} \in \mathcal{C}^{n_{\mathrm{d}} \times 1}$ to recover the source signal $s$. To this end, the detected source symbol $\hat{s}$ can be expressed as

$$
\hat{s}=\mathbf{w}_{\mathrm{d}}^{H} \mathbf{y}_{\mathrm{d}, q_{\mathrm{o}}} .
$$

Substituting (1) and (2) into (3), and taking mathematical expectation over independent realizations of signal $s$ and noise components, the SNR at the destination can be expressed as

$$
\gamma^{\left(q_{\mathrm{o}}\right)}=\frac{c_{\mathrm{s}, q_{\mathrm{o}}} c_{\mathrm{d}, q_{\mathrm{o}}}\left|\mathbf{w}_{\mathrm{d}}^{H} \mathbf{H}_{\mathrm{d}, q_{\mathrm{o}}} \mathbf{Z}_{q_{\mathrm{o}}} \mathbf{H}_{\mathrm{s}, q_{\mathrm{o}}} \mathbf{w}_{\mathrm{t}}\right|^{2}}{\sigma_{n_{\mathrm{r}}}^{2} c_{\mathrm{d}, q_{\mathrm{o}}}\left\|\mathbf{w}_{\mathrm{d}}^{H} \mathbf{H}_{\mathrm{d}, q_{\mathrm{o}}} \mathbf{Z}_{q_{\mathrm{o}}}\right\|^{2}+\sigma_{n_{\mathrm{d}}}^{2} \mathbf{w}_{\mathrm{d}}^{H} \mathbf{w}_{\mathrm{d}}} .
$$

With the help of (1), the instantaneous transmit power of the $q_{\mathrm{o}}$ th relay can be given by

$\mathrm{E}\left\{\left(\mathbf{Z}_{q_{\mathrm{o}}} \mathbf{y}_{\mathrm{r}, q_{\mathrm{o}}}\right)^{H}\left(\mathbf{Z}_{q_{\mathrm{o}}} \mathbf{y}_{\mathrm{r}, q_{\mathrm{o}}}\right)\right\}=c_{\mathrm{s}, q_{\mathrm{o}}}\left\|\mathbf{Z}_{q_{\mathrm{o}}} \mathbf{H}_{\mathrm{s}, q_{\mathrm{o}}} \mathbf{w}_{\mathrm{t}}\right\|^{2}+\sigma_{n_{\mathrm{r}}}^{2}\left\|\mathbf{Z}_{q_{\mathrm{o}}}\right\|^{2}$

where the expectation is only taken with respect to signal and noise realizations. Let $P_{\mathrm{R}, q_{\mathrm{o}}}$ and $P_{\mathrm{S}}$ be the maximum transmit powers available at the $q_{\mathrm{o}}$ th relay and source, respectively. The optimal source and destination beamforming vectors $\mathbf{w}_{\mathrm{t}}$ and $\mathbf{w}_{\mathrm{d}}$, respectively, and the AF processing matrix $\mathbf{Z}_{q_{\circ}}$ can be obtained by solving the problem of maximizing the destination-SNR (4) under the source power constraint $\left\|\mathbf{w}_{\mathrm{t}}\right\|^{2} \leq P_{\mathrm{S}}$ and the relay power constraint $c_{\mathrm{s}, q_{\mathrm{o}}}\left\|\mathbf{Z}_{q_{\mathrm{o}}} \mathbf{H}_{\mathrm{s}, q_{\mathrm{o}}} \mathbf{w}_{\mathrm{t}}\right\|^{2}+\sigma_{n_{\mathrm{r}}}^{2}\left\|\mathbf{Z}_{q_{\mathrm{o}}}\right\|^{2} \leq P_{\mathrm{R}, q_{\mathrm{o}}}$. It is well known that the optimal $\mathbf{Z}_{q_{\mathrm{o}}}, \mathbf{w}_{\mathrm{t}}$ and $\mathbf{w}_{\mathbf{d}}$ are those for which both the links from the source to the selected relay and from the selected relay to the destination form MRC plus maximum-ratio transmitting system [23]. This means that $\mathbf{w}_{\mathrm{t}}$ and $\mathbf{w}_{\mathrm{d}}$ should be matched to the $\mathcal{S}-\mathcal{R}_{q_{\mathrm{o}}}$ and $\mathcal{R}_{q_{\mathrm{o}}}-\mathcal{D}$ channels, respectively, and $\mathbf{Z}_{q_{\circ}}$ should be matched to both the $\mathcal{S}-\mathcal{R}_{q_{\mathrm{o}}}$ and $\mathcal{R}_{q_{\mathrm{o}}}-\mathcal{D}$

\footnotetext{
${ }^{3}$ For notational convenience, the variance of relay noise is assumed to be the same for all relay nodes.
}

channels. Thus, the optimal $\mathbf{Z}_{q_{\mathrm{o}}}, \mathbf{w}_{\mathrm{t}}$, and $\mathbf{w}_{\mathrm{d}}$ have the following structure [23]:

$$
\mathbf{Z}_{q_{\mathrm{o}}}=\sqrt{a}_{q_{\mathrm{o}}} \mathbf{v}_{\mathrm{d}, q_{\mathrm{o}}}^{1}\left(\mathbf{u}_{\mathrm{s}, q_{\mathrm{o}}}^{1}\right)^{H}, \quad \mathbf{w}_{\mathrm{t}}=\sqrt{b} \mathbf{v}_{\mathrm{s}, q_{\mathrm{o}}}^{1}, \quad \mathbf{w}_{\mathrm{d}}=\mathbf{u}_{\mathrm{d}, q_{\mathrm{o}}}^{1}
$$

where $a_{q_{\mathrm{o}}} \geq 0$ and $b \geq 0$ are the scaling factors that control the relay and source powers, $\mathbf{u}_{\mathrm{d}, q_{\mathrm{o}}}^{1} / \mathbf{v}_{\mathrm{d}, q_{\mathrm{o}}}^{1}$ and $\mathbf{u}_{\mathrm{s}, q_{\mathrm{o}}}^{1} / \mathbf{v}_{\mathrm{s}, q_{\mathrm{o}}}^{1}$ are the left/right singular vectors corresponding to the largest singular values of $\mathbf{H}_{\mathrm{d}, q_{\mathrm{o}}}$ and $\mathbf{H}_{\mathrm{s}, q_{\mathrm{o}}}$, respectively. From (6), the rank-one matrix $\mathbf{Z}_{q_{\mathrm{o}}}$ can be viewed as a product of receive and transmit beamformers of the $q_{\mathrm{o}}$ th relay. Substituting (6) into (4), and solving for $a$ and $b$, the destination-SNR can be expressed as

$$
\begin{aligned}
\gamma^{\left(q_{\mathrm{o}}\right)} & =\frac{P_{\mathrm{S}} P_{\mathrm{R}, q_{\mathrm{o}}} c_{\mathrm{d}, q_{\mathrm{o}}} c_{\mathrm{s}, q_{\mathrm{o}}} \alpha_{\mathrm{d}, q_{\mathrm{o}}} \alpha_{\mathrm{s}, q_{\mathrm{o}}}}{P_{\mathrm{R}, q_{\mathrm{o}}} c_{\mathrm{d}, q_{\mathrm{o}}} \sigma_{n_{\mathrm{r}}}^{2} \alpha_{\mathrm{d}, q_{\mathrm{o}}}+P_{\mathrm{S}} c_{\mathrm{s}, q_{\mathrm{o}}} \sigma_{n_{\mathrm{d}}}^{2} \alpha_{\mathrm{s}, q_{\mathrm{o}}}+\sigma_{n_{\mathrm{d}}}^{2} \sigma_{n_{\mathrm{r}}}^{2}} \\
& \triangleq \frac{e_{\mathrm{d}, q_{\mathrm{o}}} \alpha_{\mathrm{d}, q_{\mathrm{o}}} g_{\mathrm{s}, q_{\mathrm{o}}} \alpha_{\mathrm{s}, q_{\mathrm{o}}}}{e_{\mathrm{d}, q_{\mathrm{o}}} \alpha_{\mathrm{d}, q_{\mathrm{o}}}+g_{\mathrm{s}, q_{\mathrm{o}}} \alpha_{\mathrm{s}, q_{\mathrm{o}}}+1}
\end{aligned}
$$

where $\alpha_{\mathrm{d}, q_{\mathrm{o}}}=\left(\sigma_{\mathrm{d}, q_{\mathrm{o}}}^{1}\right)^{2}$ and $\alpha_{\mathrm{s}, q_{\mathrm{o}}}=\left(\sigma_{\mathrm{s}, q_{\mathrm{o}}}^{1}\right)^{2}$ are the largest eigenvalues of $\mathbf{H}_{\mathrm{d}, q_{\mathrm{o}}}^{H} \mathbf{H}_{\mathrm{d}, q_{\mathrm{o}}}$ and $\mathbf{H}_{\mathrm{s}, q_{\mathrm{o}}}^{H} \mathbf{H}_{\mathrm{s}, q_{\mathrm{o}}}$, respectively, $e_{\mathrm{d}, q_{\mathrm{o}}}=$ $\frac{c_{\mathrm{d}, q_{\mathrm{o}}} P_{\mathrm{R}, q_{\mathrm{o}}}}{\sigma_{n_{\mathrm{d}}}^{2}}$, and $g_{\mathrm{s}, q_{\mathrm{o}}}=\frac{c_{\mathrm{s}, q_{\mathrm{o}}} P_{\mathrm{S}}}{\sigma_{n_{\mathrm{r}}}^{2}}$. Note that, in the above equation, $e_{\mathrm{d}, q_{\circ}}$ and $g_{\mathrm{s}, q_{\circ}}$ are the average SNRs of the $\mathcal{R}_{q_{\circ}}-\mathcal{D}$ and $\mathcal{S}-\mathcal{R}_{q_{\circ}}$ links, respectively. The PDFs of $\left\{\alpha_{\mathrm{s}, q}\right\}_{q=1}^{2}$ and $\left\{\alpha_{\mathrm{d}, q}\right\}_{q=1}^{2}$ are given by [29]

$$
\begin{aligned}
& f_{\alpha_{\mathrm{s}, q}}(x)=\sum_{i_{q}=1}^{a_{1, q}} \sum_{m_{q}=b_{1, q}}^{b_{2, q}} d_{i_{q}, m_{q}} i_{q}^{m_{q}+1} \frac{x^{m_{q}} \mathrm{e}^{-i_{q} x}}{m_{q} !} \\
& f_{\alpha_{\mathrm{d}, q}}(x)=\sum_{j_{q}=1}^{a_{2, q}} \sum_{n_{q}=c_{1, q}}^{c_{2, q}} d_{j_{q}, n_{q}} j_{q}^{n_{q}+1} \frac{x^{n_{q}} \mathrm{e}^{-j_{q} x}}{n_{q} !}
\end{aligned}
$$

where the coefficients $d_{i_{q}, m_{q}}$ and $d_{j_{q}, n_{q}}$ have been calculated for different MIMO antenna configurations (see [29, Tables I-IV]), and for notational convenience, we have defined $a_{1, q} \triangleq \min \left(n_{\mathrm{s}}, n_{\mathrm{r}, q}\right), b_{1, q} \triangleq\left|n_{\mathrm{s}}-n_{\mathrm{r}, q}\right|, b_{2, q} \triangleq$ $\left(n_{\mathrm{s}}+n_{\mathrm{r}, q}\right) i_{q}-2 i_{q}^{2}, a_{2, q} \triangleq \min \left(n_{\mathrm{d}}, n_{\mathrm{r}, q}\right), c_{1, q} \triangleq\left|n_{\mathrm{d}}-n_{\mathrm{r}, q}\right|$ and $c_{2, q} \triangleq\left(n_{\mathrm{d}}+n_{\mathrm{r}, q}\right) j_{q}-2 j_{q}^{2}$. Note that, according to [29],

$$
\sum_{i_{q}=1}^{a_{1, q}} \sum_{m_{q}=b_{1, q}}^{b_{2, q}} d_{i_{q}, m_{q}}=1, \quad \sum_{j_{q}=1}^{a_{2, q}} \sum_{n_{q}=c_{1, q}}^{c_{2, q}} d_{j_{q}, n_{q}}=1 .
$$

Let $\gamma$ be the instantaneous SNR at the destination. The outage probability at the destination is defined as

$$
P_{\text {out }}=\operatorname{Pr}\left\{\gamma \leq \gamma_{\mathrm{th}}\right\}
$$

where $\gamma_{\mathrm{th}}$ represents the quality of service (QoS) requirement at the destination and is determined according to system specifications. According to the proposed data transmission protocol, only one relay is active during the given two-time-slot period. The destination observes $\gamma^{(1)}$ if relay $q_{\mathrm{o}}=1$ is selected, whereas the destination observes $\gamma^{(2)}$ if the relay $q_{\mathrm{o}}=2$ is selected. Mathematically, the index of the selected relay can be described as

$$
q_{\mathrm{o}}= \begin{cases}1, & \text { if } \alpha_{\mathrm{s}, 1} g_{\mathrm{s}, 1} \geq \alpha_{\mathrm{s}, 2} g_{\mathrm{s}, 2} \\ 2, & \text { if } \alpha_{\mathrm{s}, 1} g_{\mathrm{s}, 1}<\alpha_{\mathrm{s}, 2} g_{\mathrm{s}, 2}\end{cases}
$$

whereas the outage probability of (10) can be expressed as

$$
\begin{aligned}
P_{\text {out }}= & \operatorname{Pr}\left\{\gamma^{\left(q_{\mathrm{o}}\right)} \leq\left.\gamma_{\mathrm{th}}\right|_{q_{\mathrm{o}}=1}\right\} \operatorname{Pr}\left\{q_{\mathrm{o}}=1\right\} \\
& +\operatorname{Pr}\left\{\gamma^{\left(q_{\mathrm{o}}\right)} \leq\left.\gamma_{\mathrm{th}}\right|_{q_{\mathrm{o}}}=2\right\} \operatorname{Pr}\left\{q_{\mathrm{o}}=2\right\} \\
= & \operatorname{Pr}\left\{\gamma^{\left(q_{\mathrm{o}}\right)} \leq \gamma_{\mathrm{th}}, q_{\mathrm{o}}=1\right\}+\operatorname{Pr}\left\{\gamma^{\left(q_{\mathrm{o}}\right)} \leq \gamma_{\mathrm{th}}, q_{\mathrm{o}}=2\right\} .
\end{aligned}
$$


It is clear from (7) and (11) that the random events $\mathcal{A}\left(\gamma^{(1)} \leq \gamma_{\text {th }}\right.$ or $\left.\gamma^{(2)} \leq \gamma_{\mathrm{th}}\right)$ and $\mathcal{B}\left(q_{\mathrm{o}}=1\right.$ or $\left.q_{\mathrm{o}}=2\right)$ are statistically dependent. As a result, the computation of $\operatorname{Pr}\{\mathcal{A}, \mathcal{B}\}$ in (12) becomes difficult. In a special case where the source can act as a global router with the availability of the instantaneous CSI of all $\mathcal{S}-\mathcal{R}_{q}$ and $\mathcal{R}_{q}-\mathcal{D}$ channels, the outage probability reduces to the product of the outage probabilities of the two-hop links. In this case (i.e., the OR scheme), the routing at the source will be based on the maximum of the SNRs of the links via relays. However, in the present analysis, we consider practical scenario where the source has CSI of only its local channels, i.e., the source does not have the CSI of any of the $\mathcal{R}_{q}-\mathcal{D}$ channels.

\section{PERFORMANCE ANALYSIS}

In this section, we analytically characterize the criterion the source employs for its route selection and derive the expression for outage probability at the destination. Since the source node has the instantaneous CSI of only its local channels, the source chooses its beamformer based on the instantaneous SNRs of channels from the source to relays. In particular, if the SNR of the $\mathcal{S}-\mathcal{R}_{1}$ channel is greater than that of the $\mathcal{S}-\mathcal{R}_{2}$ channel, the source matches its beamformer to the $\mathcal{S}-\mathcal{R}_{1}$ channel, i.e., $\mathbf{w}_{\mathrm{t}}=\sqrt{P}_{\mathrm{S}} \mathbf{v}_{\mathrm{s}, 1}^{1}$ is applied. If the SNR of the $\mathcal{S}-\mathcal{R}_{2}$ channel is greater than that of the $\mathcal{S}-\mathcal{R}_{1}$ channel, the source uses the beamformer $\mathbf{w}_{\mathrm{t}}=\sqrt{P}_{\mathrm{S}} \mathbf{v}_{\mathrm{s}, 2}^{1}$. The routing at the source is based only on its local CSI and obviously, the knowledge of channels from relays to the destination is not assumed. Substituting (7) and (11) into (12) and after some simple steps, we can obtain

$$
\begin{aligned}
P_{\mathrm{out}}=\left[I \triangleq \operatorname { P r } \left\{g_{\mathrm{s}, 1} \alpha_{\mathrm{s}, 1}\left(e_{\mathrm{d}, 1} \alpha_{\mathrm{d}, 1}-\gamma_{\mathrm{th}}\right) \leq \gamma_{\mathrm{th}}\right.\right. \\
\left.\left.\times\left(e_{\mathrm{d}, 1} \alpha_{\mathrm{d}, 1}+1\right), \alpha_{\mathrm{s}, 1} \geq d_{\mathrm{c}} \alpha_{\mathrm{s}, 2}\right\}\right] \\
+\left[I I \triangleq \operatorname { P r } \left\{g_{\mathrm{s}, 2} \alpha_{\mathrm{s}, 2}\left(e_{\mathrm{d}, 2} \alpha_{\mathrm{d}, 2}-\gamma_{\mathrm{th}}\right) \leq \gamma_{\mathrm{th}}\right.\right. \\
\left.\left.\times\left(e_{\mathrm{d}, 2} \alpha_{\mathrm{d}, 2}+1\right), \alpha_{\mathrm{s}, 2} \geq \tilde{d}_{\mathrm{c}} \alpha_{\mathrm{s}, 1}\right\}\right]
\end{aligned}
$$

where $d_{\mathrm{c}} \triangleq \frac{g_{\mathrm{s}, 2}}{g_{\mathrm{s}, 1}}$ and $\tilde{d}_{\mathrm{c}} \triangleq \frac{1}{d_{\mathrm{c}}}$. In order to derive the closed-form expression for the outage probability (13), we have to determine the joint probabilities $I$ and $I I$ in (13). However, it is clear that the derivations of closed-form expression for $I I$ follow similarly to that of $I$. In the following, we focus on the term $I$. Notice that $\alpha_{\mathrm{s}, 1}, \alpha_{\mathrm{s}, 2}$ and $\alpha_{\mathrm{d}, 1}$ are statistically independent continuous random variables. As can be seen from (13), however, the events that make the joint probability $I$ of (13) are not independent. The two events of $I$ are dependent through the same random variable $\alpha_{\mathrm{s}, 1}$. Integrating over random variables $\tilde{\alpha}_{\mathrm{s}, 2}=d_{\mathrm{c}} \alpha_{\mathrm{s}, 2}$ and $\alpha_{\mathrm{d}, 1}$, we can express $I$ as

$$
\begin{aligned}
I= & \int_{0}^{\infty} \int_{0}^{y_{u}(z)} \operatorname{Pr}\left\{\mathcal { A } _ { 1 } \triangleq \left[g_{\mathrm{s}, 1} \alpha_{\mathrm{s}, 1}\left(e_{\mathrm{d}, 1} z-\gamma_{\mathrm{th}}\right)\right.\right. \\
& \left.\left.\leq \gamma_{\mathrm{th}}\left(e_{\mathrm{d}, 1} z+1\right)\right], \alpha_{\mathrm{s}, 1} \geq y\right\} f_{\tilde{\alpha}_{\mathrm{s}, 2}}(y) d y f_{\alpha_{\mathrm{d}, 1}}(z) d z
\end{aligned}
$$

where $y_{u}(z)$ is the upper limit for $y$ and it depends on $z$. It is clear that, for $0 \leq z \leq \frac{\gamma_{\text {th }}}{e_{\mathrm{d}, 1}}$, the event $\mathcal{A}_{1}$ is certain (i.e., the probability of its occurrence is 1$)$. In this case, $\operatorname{Pr}\left\{\mathcal{A}_{1}, \alpha_{\mathrm{s}, 1} \geq\right.$ $y\}$ in (14) reduces to $\operatorname{Pr}\left\{\alpha_{\mathrm{s}, 1} \geq y\right\}$ and the upper limit for $y$ becomes independent of $z$. As a result, for $0 \leq z \leq \frac{\gamma_{\mathrm{th}}}{e_{\mathrm{d}, 1}}$, (14) can be written as

$$
\begin{aligned}
{[I]_{z=0}^{\frac{\gamma_{\mathrm{th}}}{\mathrm{d}_{1} 1}} } & =\int_{0}^{\frac{\gamma_{\mathrm{th}}}{e_{\mathrm{d}, 1}}} \int_{0}^{\infty} \operatorname{Pr}\left\{\alpha_{\mathrm{s}, 1} \geq y\right\} f_{\tilde{\alpha}_{\mathrm{s}, 2}}(y) d y f_{\alpha_{\mathrm{d}, 1}}(z) d z \\
& \triangleq \operatorname{Pr}\left\{\alpha_{\mathrm{s}, 1} \geq \tilde{\alpha}_{\mathrm{s}, 2}\right\} \int_{0}^{\frac{\gamma_{\mathrm{th}}}{e_{\mathrm{d}, 1}}} f_{\alpha_{\mathrm{d}, 1}}(z) d z .
\end{aligned}
$$

For the case $\frac{\gamma_{\mathrm{th}}}{e_{\mathrm{d}, 1}}<z \leq \infty, e_{\mathrm{d}, 1} z-\gamma_{\mathrm{th}}>0$, and thus, (14) can be rewritten as

$$
\begin{aligned}
{[I]_{z=\frac{\gamma_{\mathrm{th}}}{\epsilon_{\mathrm{d}, 1}}}^{\infty}=\int_{\frac{\gamma_{\mathrm{th}}}{e_{\mathrm{d}, 1}}}^{\infty} \int_{0}^{y_{u}(z)} \operatorname{Pr}\left\{\alpha_{\mathrm{s}, 1}\right.} & \left.\leq \frac{\gamma_{\mathrm{th}}\left(e_{\mathrm{d}, 1} z+1\right)}{g_{\mathrm{s}, 1}\left(e_{\mathrm{d}, 1} z-\gamma_{\mathrm{th}}\right)}, \alpha_{\mathrm{s}, 1} \geq y\right\} \\
& \times f_{\tilde{\alpha}_{\mathrm{s}, 2}}(y) d y f_{\alpha_{\mathrm{d}, 1}}(z) d z .
\end{aligned}
$$

In the remaining part of this section, we derive closed-form expressions for (15) and (16). To this end, (15) is expressed in closed-form (see Appendix A) as

$$
\begin{aligned}
{[I]_{z=0}^{\frac{\gamma_{\mathrm{th}}}{e_{\mathrm{d}, 1}}=} } & \left\{1-\sum_{i_{1}=1}^{a_{1,1}} \sum_{m_{1}=b_{1,1}}^{b_{2,1}} \sum_{i_{2}=1}^{a_{1,2}} \sum_{m_{2}=b_{1,2}}^{b_{2,2}} \frac{\Gamma\left(m_{1}+m_{2}+2\right)}{m_{1} ! m_{2} !}\right. \\
& \times \frac{d_{i_{2}, m_{2}} d_{i_{1}, m_{1}}\left(i_{1} d_{\mathrm{c}}\right)^{m_{1}+1}}{i_{2}^{m_{1}+1}\left(m_{1}+1\right)}{ }_{2} F_{1}\left(m_{1}+m_{2}+2\right. \\
& \left.\left.m_{1}+1 ; m_{1}+2 ;-\frac{i_{1} d_{\mathrm{c}}}{i_{2}}\right)\right\} \\
& \times \sum_{j_{1}=1}^{a_{2,1}} \sum_{n_{1}=c_{1,1}}^{c_{2,1}} \frac{d_{j_{1}, n_{1}}}{n_{1} !} \gamma_{\mathrm{L}}\left(n_{1}+1, \frac{j_{1} \gamma_{\mathrm{th}}}{e_{\mathrm{d}, 1}}\right)
\end{aligned}
$$

where $\gamma_{\mathbf{L}}(\cdot, \cdot)$ is the lower incomplete Gamma function and ${ }_{2} F_{1}()$ is the Gauss hypergeometric function [30]. With the derivations of Appendix B, we can express (16) as

$$
\begin{aligned}
& {[I]_{z=\frac{\gamma_{\mathrm{th}}}{e_{\mathrm{d}, 1}}=} } \sum_{i_{2}=1}^{a_{1,2}} \sum_{m_{2}=b_{1,2}}^{b_{2,2}} \sum_{i_{1}=1}^{a_{1,1}} \sum_{m_{1}=b_{1,1}}^{b_{2,1}} \frac{d_{i_{2}, m_{2}} d_{i_{1}, m_{1}}}{m_{2} !} \\
& \times\left\{-\sum_{q=0}^{m_{1}} \frac{1}{q !} \mathrm{e}^{-\frac{i_{1} \gamma_{\mathrm{th}}}{g_{\mathrm{s}, 1}}\left(\frac{i_{1} \gamma_{\mathrm{th}}}{g_{\mathrm{s}, 1}}\right)^{q} \int_{0}^{\infty} \bar{z}^{-q}\left(\bar{z}+\gamma_{\mathrm{th}}+1\right)^{q}}\right. \\
& \times \gamma_{\mathrm{L}}\left(m_{2}+1, \frac{i_{2} \gamma_{\mathrm{th}}}{d_{\mathrm{c}} g_{\mathrm{s}, 1}}\left[1+\frac{\gamma_{\mathrm{th}}+1}{\bar{z}}\right]\right) \mathrm{e}^{-i_{1} \gamma_{\mathrm{th}}\left(g_{\mathrm{s}, 1} \bar{z}\right)^{-1}} \\
& \times \mathrm{e}^{-i_{1} \gamma_{\mathrm{th}}^{2}\left(g_{\mathrm{s}, 1} \bar{z}\right)^{-1}} f_{\alpha_{\mathrm{d}, 1}}\left(\frac{\bar{z}+\gamma_{\mathrm{th}}}{e_{\mathrm{d}, 1}}\right) \frac{d \bar{z}}{e_{\mathrm{d}, 1}}+\sum_{p=0}^{m_{1}} \frac{i_{1}^{p}}{p !} \\
& \times\left(\frac{i_{2}}{d_{\mathrm{c}}}\right)^{m_{2}+1}\left(i_{1}+\frac{i_{2}}{d_{\mathrm{c}}}\right)^{-\left(m_{2}+p+1\right)} \\
& \times \int_{0}^{\infty} \gamma_{\mathrm{L}}\left(m_{2}+p+1,\left(i_{1}+\frac{i_{2}}{d_{\mathrm{c}}}\right) \frac{\gamma_{\mathrm{th}}}{g_{\mathrm{s}, 1}}\left[1+\frac{\gamma_{\mathrm{th}}+1}{\bar{z}}\right]\right) \\
&\left.\times f_{\alpha_{\mathrm{d}, 1}}\left(\frac{\bar{z}+\gamma_{\mathrm{th}}}{e_{\mathrm{d}, 1}}\right) \frac{1}{e_{\mathrm{d}, 1}} d \bar{z}\right\}
\end{aligned}
$$


As shown in Appendix C, the first integral of (18) can be expressed as

$$
\begin{aligned}
& I_{3}=2 m_{2} ! \sum_{j_{1}=1}^{a_{2,1}} \sum_{n_{1}=c_{1,1}}^{c_{2,1}} \frac{d_{j_{1}, n_{1}} j_{1}^{n_{1}+1}}{n_{1} ! e_{\mathrm{d}, 1}^{n_{1}+1}} \mathrm{e}^{-\frac{j_{1} \gamma_{\mathrm{th}}}{e_{\mathrm{d}, 1}}} \\
& \times\left\{\sum_{v=0}^{n_{1}} \sum_{w=0}^{q}\left(\begin{array}{c}
n_{1} \\
v
\end{array}\right)\left(\begin{array}{c}
q \\
w
\end{array}\right) \gamma_{\mathrm{th}}^{n_{1}-v}\left(\gamma_{\mathrm{th}}+1\right)^{q-w}\right. \\
& \times\left(\frac{\hat{d}_{2} e_{\mathrm{d}, 1}}{j_{1}}\right)^{\frac{v+w-q+1}{2}} K_{v+w-q+1}\left(2 \sqrt{\frac{\hat{d}_{2} j_{1}}{e_{\mathrm{d}, 1}}}\right) \\
&-\mathrm{e}^{-d_{1}} \sum_{u=0}^{m_{2}} \sum_{a=0}^{n_{1}} \sum_{b=0}^{u+q} \frac{d_{1}^{u}}{u !} \\
& \times\left(\begin{array}{c}
n_{1} \\
a
\end{array}\right)\left(\begin{array}{c}
u+q \\
b
\end{array}\right) \gamma_{\mathrm{th}}^{n_{1}-a}\left(\frac{\tilde{d}_{2} e_{\mathrm{d}, 1}}{j_{1}}\right) \\
& \times\left(\gamma_{\mathrm{th}}+1\right)^{u+q-b} K_{a+b-u-q+1}\left(2 \sqrt{\frac{a+b-u-q+1}{2}}\right.
\end{aligned}
$$

where $K_{\nu}(\cdot, \cdot)$ is the modified Bessel function of the second kind [30] with order $\nu$. Using the derivations of Appendix D, the second integral of (18) can be written as

$$
\begin{aligned}
I_{4}=( & \left.m_{2}+p\right) ! \sum_{j_{1}=1}^{a_{2,1}} \sum_{n_{1}=c_{1,1}}^{c_{2,1}} \frac{d_{j_{1}, n_{1}}}{n_{1} !} \\
\times\{ & \Gamma\left(n_{1}+1, \frac{\gamma_{\mathrm{th}} j_{1}}{e_{\mathrm{d}, 1}}\right) \\
& -2 \mathrm{e}^{-\left(c_{1}+\frac{\gamma_{\mathrm{th}} j_{1}}{e_{\mathrm{d}, 1}}\right)\left(\frac{j_{1}}{e_{\mathrm{d}, 1}}\right)^{n_{1}+1} \sum_{r=0}^{m_{2}+p} \sum_{s=0}^{n_{1}} \sum_{t=0}^{r}\left(\begin{array}{c}
n_{1} \\
s
\end{array}\right)\left(\begin{array}{c}
r \\
t
\end{array}\right)} \\
& \times \frac{c_{1}^{r}}{r !} \gamma_{\mathrm{th}}^{n_{1}-s}\left(\gamma_{\mathrm{th}}+1\right)^{r-t}\left[\frac{c_{1}\left(\gamma_{\mathrm{th}}+1\right) e_{\mathrm{d}, 1}}{j_{1}}\right]^{\frac{s+t-r+1}{2}} \\
& \left.\times K_{s+t-r+1}\left[2 \sqrt{\frac{c_{1}\left(\gamma_{\mathrm{th}}+1\right) j_{1}}{e_{\mathrm{d}, 1}}}\right]\right\}
\end{aligned}
$$

where $\Gamma(\cdot, \cdot)$ is the upper incomplete Gamma function. After substituting (19) and (20) into (18) and then using (17), the first part of the outage probability (13) is obtained. The term $I I$ of (13) can be obtained simply by interchanging $g_{\mathrm{s}, 1}$ and $g_{\mathrm{s}, 2}$ and replacing $e_{\mathrm{d}, 1}$ by $e_{\mathrm{d}, 2}$ in (17) and (18)-(20).

Remark 3: The derived closed-form expressions for different components of the overall outage probability appear to be long and complicated due to multiple summation terms. However, the upper limits for all the summation terms are finite and functions of the numbers of antennas at the source, relay, and destination. In practical applications, the number of antennas is limited and, therefore, the burden for computing the closed-form expressions is relatively low.

\section{A. Special Case With $n_{\mathrm{s}}=n_{\mathrm{r}}=n_{\mathrm{r}, 1}=n_{\mathrm{r}, 2}=1$}

For a network with single-antenna source, destination and relays, the first term $I$ of the overall outage probability of (13) can be obtained by simply substituting $n_{\mathrm{s}}=n_{\mathrm{r}}=n_{\mathrm{r}, 1}=n_{\mathrm{r}, 2}=1$ into (17) and (18)-(20). Noting that all $d_{i_{q}, m_{q}}$ and $d_{j_{q}, n_{q}}$ reduce to $d_{1,0}=1$ for this case, $I$ of (13) can be expressed as

$$
\begin{aligned}
\tilde{I}= & \left(1-{ }_{2} F_{1}\left(2,1 ; 2 ;-d_{\mathrm{c}}\right) d_{\mathrm{c}}\right) \gamma_{\mathrm{L}}\left(1, \frac{\gamma_{\mathrm{th}}}{e_{\mathrm{d}, 1}}\right) \\
& -\mathrm{e}^{-\gamma_{\mathrm{th}}\left(\frac{1}{g_{\mathrm{s}, 1}}+\frac{1}{e_{\mathrm{d}, 1}}\right)} 2\left(\frac{\tilde{\hat{d}}_{2}}{e_{\mathrm{d}, 1}}\right)^{\frac{1}{2}} K_{1}\left(\frac{4 \tilde{\hat{d}}_{2}}{e_{\mathrm{d}, 1}}\right)^{\frac{1}{2}} \\
& +\mathrm{e}^{-\gamma_{\mathrm{th}}\left(\frac{1}{g_{\mathrm{s}, 1}}+\frac{1}{e_{\mathrm{d}, 1}}+\frac{1}{g_{\mathrm{s}, 2}}\right)} 2\left(\frac{\tilde{\tilde{d}}_{2}}{e_{\mathrm{d}, 1}}\right)^{\frac{1}{2}} K_{1}\left(\frac{4 \tilde{\tilde{d}}_{2}}{e_{\mathrm{d}, 1}}\right)^{\frac{1}{2}} \\
& +\frac{\Gamma\left(1, \frac{\gamma_{\mathrm{th}}}{e_{\mathrm{d}, 1}}\right)}{d_{\mathrm{c}}+1}-\frac{1}{d_{\mathrm{c}}+1} \mathrm{e}^{-\left(\tilde{c}_{1}+\frac{\gamma_{\mathrm{th}}}{e_{\mathrm{d}, 1}}\right)} \\
& \times 2\left(\frac{\tilde{c}_{1}\left(\gamma_{\mathrm{th}}+1\right)}{e_{\mathrm{d}, 1}}\right)^{\frac{1}{2}} K_{1}\left(\frac{4 \tilde{c}_{1}\left(\gamma_{\mathrm{th}}+1\right)}{e_{\mathrm{d}, 1}}\right)^{\frac{1}{2}}
\end{aligned}
$$

where $\tilde{\hat{d}}_{2}=\frac{\gamma_{\mathrm{th}}\left(\gamma_{\mathrm{th}}+1\right)}{g_{\mathrm{s}, 1}}, \tilde{c}_{1}=\left(\frac{1}{g_{\mathrm{s}, 1}}+\frac{1}{g_{\mathrm{s}, 2}}\right) \gamma_{\mathrm{th}}, \tilde{\tilde{d}}_{2}=\tilde{c}_{1}\left(\gamma_{\mathrm{th}}+\right.$ $1)$, and the Gauss hypergeometric function ${ }_{2} F_{1}\left(2,1 ; 2 ;-d_{c}\right)$ can be shown to be equal to $\left(1+d_{\mathrm{c}}\right)^{-1}$ with the help of [30, eqs. (9.100), (1.112.1)]. Furthermore, noting that $\gamma_{\mathrm{L}}\left(1, \frac{\gamma_{\mathrm{th}}}{e_{\mathrm{d}, 1}}\right)+$ $\Gamma\left(1, \frac{\gamma_{\mathrm{th}}}{e_{\mathrm{d}, 1}}\right)=\Gamma(1)=1$, we can simplify (21) to the following form:

$$
\begin{aligned}
\tilde{I}= & \frac{1}{d_{\mathrm{c}}+1}\left\{1-\mathrm{e}^{-\left(\tilde{c}_{1}+\frac{\gamma_{\mathrm{th}}}{e_{\mathrm{d}, 1}}\right)} 2\left(\frac{\tilde{c}_{1}\left(\gamma_{\mathrm{th}}+1\right)}{e_{\mathrm{d}, 1}}\right)^{\frac{1}{2}}\right. \\
& \left.\times K_{1}\left(\frac{4 \tilde{c}_{1}\left(\gamma_{\mathrm{th}}+1\right)}{e_{\mathrm{d}, 1}}\right)^{\frac{1}{2}}\right\} \\
- & \mathrm{e}^{-\gamma_{\mathrm{th}}\left(\frac{1}{g_{\mathrm{s}, 1}}+\frac{1}{e_{\mathrm{d}, 1}}\right)} 2\left(\frac{\tilde{\hat{d}}_{2}}{e_{\mathrm{d}, 1}}\right)^{\frac{1}{2}} K_{1}\left(\frac{4 \tilde{\hat{d}}_{2}}{e_{\mathrm{d}, 1}}\right)^{\frac{1}{2}} \\
& +\mathrm{e}^{-\gamma_{\mathrm{th}}\left(\frac{1}{g_{\mathrm{s}, 1}}+\frac{1}{e_{\mathrm{d}, 1}}+\frac{1}{g_{\mathrm{s}, 2}}\right)} 2\left(\frac{\tilde{\tilde{d}}_{2}}{e_{\mathrm{d}, 1}}\right)^{\frac{1}{2}} K_{1}\left(\frac{4 \tilde{\tilde{d}}_{2}}{e_{\mathrm{d}, 1}}\right)^{\frac{1}{2}} .
\end{aligned}
$$

Interchanging $g_{\mathrm{s}, 1}$ and $g_{\mathrm{s}, 2}$ in (22) and replacing $e_{\mathrm{d}, 1}$ by $e_{\mathrm{d}, 2}$, we can obtain the second term $I I$ of the outage probability (13) for the case $n_{\mathrm{s}}=n_{\mathrm{r}}=n_{\mathrm{r}, 1}=n_{\mathrm{r}, 2}=1$. Summing the resulting expression with (22), using the definitions $\tilde{\alpha} \triangleq \frac{1}{g_{\mathrm{s}, 1}}=\frac{1}{g_{\mathrm{s}, 2}}, \tilde{\beta} \triangleq$ $\frac{1}{e_{\mathrm{d}, 1}}=\frac{1}{e_{\mathrm{d}, 2}}$, and making the approximation that $\gamma_{\mathrm{th}}+1 \approx \gamma_{\mathrm{th}}$, we can obtain the following result for outage probability:

$$
\begin{aligned}
\tilde{P}_{\text {out }}=1- & 2 \mathrm{e}^{-(\tilde{\alpha}+\tilde{\beta}) \gamma_{\mathrm{th}}} 2 \gamma_{\mathrm{th}}(\tilde{\alpha} \tilde{\beta})^{\frac{1}{2}} K_{1}\left(4 \gamma_{\mathrm{th}}^{2} \tilde{\alpha} \tilde{\beta}\right)^{\frac{1}{2}} \\
& +\mathrm{e}^{-(2 \tilde{\alpha}+\tilde{\beta}) \gamma_{\mathrm{th}}} 2 \gamma_{\mathrm{th}}(2 \tilde{\alpha} \tilde{\beta})^{\frac{1}{2}} K_{1}\left(4 \gamma_{\mathrm{th}}^{2} 2 \tilde{\alpha} \tilde{\beta}\right)^{\frac{1}{2}}
\end{aligned}
$$

which is same as the outage probability expression derived in [18, eq. (2)]. For $g_{\mathrm{s}, 2}=0$, we find that (21) reduces to the following form:

$$
\tilde{\tilde{I}}=1-\mathrm{e}^{-\gamma_{\mathrm{th}}\left(\frac{1}{g_{\mathrm{s}, 1}}+\frac{1}{e_{\mathrm{d}, 1}}\right)} 2\left(\frac{\tilde{\hat{d}}_{2}}{e_{\mathrm{d}, 1}}\right)^{\frac{1}{2}} K_{1}\left(\frac{4 \tilde{\hat{d}}_{2}}{e_{\mathrm{d}, 1}}\right)^{\frac{1}{2}}
$$

which is the outage probability of the two-hop link corresponding to the first relay when each node is equipped with a single antenna. For the approximation $\gamma_{\mathrm{th}}+1 \approx \gamma_{\mathrm{th}},(24)$ is the same as the outage probability expression derived in [31, eq. (27)]. In (21), after replacing $e_{\mathrm{d}, 1}$ by $e_{\mathrm{d}, 2}$, and interchanging $g_{\mathrm{s}, 1}$ and $g_{\mathrm{s}, 2}$, we can get the second term $I I$ of (13). In this 
case, the resulting expression for $g_{\mathrm{s}, 1}=0$, also reduces to the similar form like that of (24) with $g_{\mathrm{s}, 1}$ and $e_{\mathrm{d}, 1}$ replaced by $g_{\mathrm{s}, 2}$ and $e_{\mathrm{d}, 2}$, respectively. Consequently, such expression gives the outage probability of the two-hop link corresponding to the second relay, where again all terminals are single-antenna nodes.

\section{ASYMPTOTIC ANALYSIS}

It is rather difficult to obtain the diversity order for the proposed selection scheme from the outage probability expressions derived in Section III. These expressions are derived from the exact PDFs $f_{\alpha_{\mathrm{s}, q}}(x)$ and $f_{\alpha_{\mathrm{d}, q}}(x)$ of (8). Notice that, for large values of $\left\{e_{\mathrm{d}, q}\right\}_{q=1}^{2}$ and $\left\{g_{\mathrm{s}, q}\right\}_{q=1}^{2}$, the outage probability (also the symbol error rate [32]) is dominated by the small values of $\gamma^{(1)}$ and $\gamma^{(2)}$ [33]. This gives rise to the importance of examining the behaviors of $f_{\alpha_{\mathrm{s}, q}}(x)$ and $f_{\alpha_{\mathrm{d}, q}}(x)$ at small values of $x$. According to [33], as $x \rightarrow 0$, the asymptotic behaviors of $f_{\alpha_{s, q}}(x)$ and $f_{\alpha_{\mathrm{d}, q}}(x)$ can be characterized by [cf. [34] for the use of similar asymptotic expression in a different application with a MIMO relay]

$f_{\alpha_{\mathrm{s}, q}}(x) \approx \frac{\mu_{\mathrm{s}, q} \prod_{k=0}^{a_{1, q}-1} k !}{\prod_{k=0}^{a_{1, q}-1}\left(t_{\mathrm{s}, q}+k\right) !} x^{\mu_{\mathrm{s}, q}-1} \triangleq r_{\mathrm{s}, q} x^{\mu_{\mathrm{s}, q}-1}$

$f_{\alpha_{\mathrm{d}, q}}(x) \approx \frac{\mu_{\mathrm{d}, q} \prod_{k=0}^{a_{2, q}-1} k !}{\prod_{k=0}^{a_{2, q}-1}\left(t_{\mathrm{d}, q}+k\right) !} x^{\mu_{\mathrm{d}, q}-1} \triangleq r_{\mathrm{d}, q} x^{\mu_{\mathrm{d}, q}-1}, \forall q$

where $\mu_{\mathrm{s}, q}=n_{\mathrm{s}} n_{\mathrm{r}, q}, \mu_{\mathrm{d}, q}=n_{\mathrm{d}} n_{\mathrm{r}, q}, t_{\mathrm{s}, q}=\max \left(n_{\mathrm{s}}, n_{\mathrm{r}, q}\right)$, $t_{\mathrm{d}, q}=\max \left(n_{\mathrm{d}}, n_{\mathrm{r}, q}\right)$, for $q=1,2$. In order to obtain the asymptotic expression for $I$ in (13), we apply the following upper bound [35]:

$$
\begin{aligned}
\frac{e_{\mathrm{d}, 1} \alpha_{\mathrm{d}, 1} g_{\mathrm{s}, 1} \alpha_{\mathrm{s}, 1}}{e_{\mathrm{d}, 1} \alpha_{\mathrm{d}, 1}+g_{\mathrm{s}, 1} \alpha_{\mathrm{s}, 1}+1} & \approx \frac{e_{\mathrm{d}, 1} \alpha_{\mathrm{d}, 1} g_{\mathrm{s}, 1} \alpha_{\mathrm{s}, 1}}{e_{\mathrm{d}, 1} \alpha_{\mathrm{d}, 1}+g_{\mathrm{s}, 1} \alpha_{\mathrm{s}, 1}} \\
& \leq \min \left(e_{\mathrm{d}, 1} \alpha_{\mathrm{d}, 1}, g_{\mathrm{s}, 1} \alpha_{\mathrm{s}, 1}\right)
\end{aligned}
$$

which is accurate for medium to high values of $e_{\mathrm{d}, 1}$ and $g_{\mathrm{s}, 1}$. With the help of (26), $I$ of (13) can be approximated for large values of $e_{\mathrm{d}, 1}$ and $g_{\mathrm{s}, 1}$ as

$$
\begin{aligned}
I \approx\left[I _ { \mathrm { e } } \triangleq \operatorname { P r } \left\{\min \left(e_{\mathrm{d}, 1} \alpha_{\mathrm{d}, 1}, g_{\mathrm{s}, 1} \alpha_{\mathrm{s}, 1}\right)\right.\right. & \\
& \left.\left.\leq \gamma_{\mathrm{th}}, g_{\mathrm{s}, 1} \alpha_{\mathrm{s}, 1} \geq g_{\mathrm{s}, 2} \alpha_{\mathrm{s}, 2}\right\}\right] .
\end{aligned}
$$

The joint probability in the above equation consists of statistically dependent random events characterized by three random variables. Computing such probability demands dealing with multidimensional integrals. Our main objective of this part is to obtain the closed-form expression of $I_{\mathrm{e}}$ based on (25). The key steps involved are rewriting (27) as the sum of two joint probability terms, transformation into multidimensional integration problems, and solution by selecting appropriate integral limits.

In (27), we know that $\min \left(e_{\mathrm{d}, 1} \alpha_{\mathrm{d}, 1}, g_{\mathrm{s}, 1} \alpha_{\mathrm{s}, 1}\right)=e_{\mathrm{d}, 1} \alpha_{\mathrm{d}, 1}$ if $e_{\mathrm{d}, 1} \alpha_{\mathrm{d}, 1} \leq g_{\mathrm{s}, 1} \alpha_{\mathrm{s}, 1}$ and $\min \left(e_{\mathrm{d}, 1} \alpha_{\mathrm{d}, 1}, g_{\mathrm{s}, 1} \alpha_{\mathrm{s}, 1}\right)=g_{\mathrm{s}, 1} \alpha_{\mathrm{s}, 1}$ if $e_{\mathrm{d}, 1} \alpha_{\mathrm{d}, 1}>g_{\mathrm{s}, 1} \alpha_{\mathrm{s}, 1}$. Thus, using the total law of probability and Bayes' theorem [36], (27) can be expressed as the following sum of two probabilities:

$$
\begin{gathered}
I_{\mathrm{e}}=\operatorname{Pr}\left\{e_{\mathrm{d}, 1} \alpha_{\mathrm{d}, 1} \leq \gamma_{\mathrm{th}}, g_{\mathrm{s}, 1} \alpha_{\mathrm{s}, 1} \geq g_{\mathrm{s}, 2} \alpha_{\mathrm{s}, 2}, e_{\mathrm{d}, 1} \alpha_{\mathrm{d}, 1} \leq g_{\mathrm{s}, 1} \alpha_{\mathrm{s}, 1}\right\} \\
+\operatorname{Pr}\left\{g_{\mathrm{s}, 1} \alpha_{\mathrm{s}, 1} \leq \gamma_{\mathrm{th}}, g_{\mathrm{s}, 1} \alpha_{\mathrm{s}, 1} \geq g_{\mathrm{s}, 2} \alpha_{\mathrm{s}, 2}, g_{\mathrm{s}, 1} \alpha_{\mathrm{s}, 1}\right. \\
\left.\quad \leq e_{\mathrm{d}, 1} \alpha_{\mathrm{d}, 1}\right\} .
\end{gathered}
$$

In Appendix E, we have proven that the first part of (28) can be expressed as

$$
\begin{aligned}
I_{\mathrm{e}, 1} & =\frac{r_{\mathrm{d}, 1} \gamma_{\mathrm{th}}^{\mu_{\mathrm{d}, 1}}}{\mu_{\mathrm{d}, 1} \mu_{\mathrm{d}, 1}} \operatorname{Pr}\left\{g_{\mathrm{s}, 1} \alpha_{\mathrm{s}, 1} \geq g_{\mathrm{s}, 2} \alpha_{\mathrm{s}, 2}\right\}-\frac{r_{\mathrm{s}, 2} r_{\mathrm{d}, 1} r_{\mathrm{s}, 1}}{\mu_{\mathrm{s}, 2}} \\
& \times \frac{\gamma_{\mathrm{th}}^{\mu_{\mathrm{s}, 2}}+\mu_{\mathrm{d}, 1}+\mu_{\mathrm{s}, 1}}{\left(\mu_{\mathrm{s}, 2}+\mu_{\mathrm{s}, 1}\right)\left(\mu_{\mathrm{s}, 2}+\mu_{\mathrm{d}, 1}+\mu_{\mathrm{s}, 1}\right) g_{\mathrm{s}, 2}^{\mu_{\mathrm{s}, 2}} e_{\mathrm{d}, 1}^{\mu_{\mathrm{d}, 1}} g_{\mathrm{s}, 1}^{\mu_{\mathrm{s}, 1}}} .
\end{aligned}
$$

As shown in Appendix F, the second part of (28) can be written as

$$
\begin{aligned}
I_{\mathrm{e}, 2}=\frac{r_{\mathrm{s}, 2} r_{\mathrm{s}, 1} \gamma_{\mathrm{th}}^{\mu_{\mathrm{s}, 2}+\mu_{\mathrm{s}, 1}}}{\mu_{\mathrm{s}, 2}\left(\mu_{\mathrm{s}, 2}\right.}+ & \left.\mu_{\mathrm{s}, 1}\right) g_{\mathrm{s}, 2}^{\mu_{\mathrm{s}, 2}} g_{\mathrm{s}, 1}^{\mu_{\mathrm{s}}}-\frac{r_{\mathrm{s}, 2} r_{\mathrm{d}, 1} r_{\mathrm{s}, 1}}{\mu_{\mathrm{s}, 2} \mu_{\mathrm{d}, 1}} \\
& \times \frac{\gamma_{\mathrm{th}, 2}^{\mu_{\mathrm{d}, 1}+\mu_{\mathrm{s}, 1}}}{\left(\mu_{\mathrm{s}, 2}+\mu_{\mathrm{d}, 1}+\mu_{\mathrm{s}, 1}\right) g_{\mathrm{s}, 2}^{\mu_{\mathrm{s}, 2}} e_{\mathrm{d}, 1}^{\mu_{\mathrm{d}, 1}} g_{\mathrm{s}, 1}^{\mu_{\mathrm{s}, 1}}} .
\end{aligned}
$$

Finally, adding (29) and (30), (28) can be expressed as

$$
\begin{aligned}
& I_{\mathrm{e}}=\frac{r_{\mathrm{d}, 1} \gamma_{\mathrm{th}}^{\mu_{\mathrm{d}, 1}}}{\mu_{\mathrm{d}, 1} e_{\mathrm{d}, 1}^{\mu_{\mathrm{d}, 1}}} \operatorname{Pr}\left\{\alpha_{\mathrm{s}, 1} \geq \frac{g_{\mathrm{s}, 2} \alpha_{\mathrm{s}, 2}}{g_{\mathrm{s}, 1}}\right\}+\frac{r_{\mathrm{s}, 2} r_{\mathrm{s}, 1}}{\mu_{\mathrm{s}, 2}\left(\mu_{\mathrm{s}, 2}+\mu_{\mathrm{s}, 1}\right)} \\
& \times \frac{\gamma_{\mathrm{th}}^{\mu_{\mathrm{s}, 2}+\mu_{\mathrm{s}, 1}}}{g_{\mathrm{s}, 2}^{\mu_{\mathrm{s}, 2}} g_{\mathrm{s}, 1}^{\mu_{\mathrm{s}, 1}}}-\frac{r_{\mathrm{s}, 2} r_{\mathrm{d}, 1} r_{\mathrm{s}, 1} \gamma_{\mathrm{th}}^{\mu_{\mathrm{s}, 2}+\mu_{\mathrm{d}, 1}+\mu_{\mathrm{s}, 1}}}{\mu_{\mathrm{s}, 2} \mu_{\mathrm{d}, 1}\left(\mu_{\mathrm{s}, 2}+\mu_{\mathrm{s}, 1}\right) g_{\mathrm{s}, 2}^{\mu_{\mathrm{s}, 2}} e_{\mathrm{d}, 1}^{\mu_{\mathrm{d}, 1}} g_{\mathrm{s}, 1}^{\mu_{\mathrm{s}, 1}}} .
\end{aligned}
$$

The asymptotic expression for $I I$ of (13) can be derived using the similar steps. For the completeness of this section, the expression $I I \approx I I_{\mathrm{e}}$ is shown as

$$
\begin{aligned}
& I I_{\mathrm{e}}=\frac{r_{\mathrm{d}, 2} \gamma_{\mathrm{th}}^{\mu_{\mathrm{d}, 2}}}{\mu_{\mathrm{d}, 2} e_{\mathrm{d}, 2}^{\mu_{\mathrm{d}}}} \operatorname{Pr}\left\{\alpha_{\mathrm{s}, 2} \geq \frac{g_{\mathrm{s}, 1} \alpha_{\mathrm{s}, 1}}{g_{\mathrm{s}, 2}}\right\}+\frac{r_{\mathrm{s}, 2} r_{\mathrm{s}, 1}}{\mu_{\mathrm{s}, 1}\left(\mu_{\mathrm{s}, 2}+\mu_{\mathrm{s}, 1}\right)} \\
& \times \frac{\gamma_{\mathrm{th}}^{\mu_{\mathrm{s}, 2}+\mu_{\mathrm{s}, 1}}}{g_{\mathrm{s}, 2}^{\mu_{\mathrm{s}, 2}} g_{\mathrm{s}, 1}^{\mu_{\mathrm{s}, 1}}}-\frac{r_{\mathrm{s}, 2} r_{\mathrm{d}, 2} r_{\mathrm{s}, 1}}{\mu_{\mathrm{s}, 1} \mu_{\mathrm{d}, 2}\left(\mu_{\mathrm{s}, 2}+\mu_{\mathrm{s}, 1}\right)} \times \frac{\gamma_{\mathrm{th}, 2}^{\mu_{\mathrm{s}}+\mu_{\mathrm{d}, 2}+\mu_{\mathrm{s}, 1}}}{g_{\mathrm{s}, 2}^{\mu_{\mathrm{s}, 2}} e_{\mathrm{d}, 2}^{\mu_{\mathrm{d}, 2}} g_{\mathrm{s}, 1}^{\mu_{\mathrm{s}, 1}}}
\end{aligned}
$$

Notice that the high SNR refers to the case when $e_{\mathrm{d}, q}$, and $g_{\mathrm{s}, q}$ for $q=1,2$ assume large values. Thus, without loss of generality (w.l.o.g), consider $e_{\mathrm{d}, 1}=\bar{\gamma}, e_{\mathrm{d}, 2}=\nu_{1} \bar{\gamma}, g_{\mathrm{s}, 1}=\nu_{2} \bar{\gamma}$, $g_{\mathrm{s}, 2}=\nu_{3} \bar{\gamma}$, i.e., the average SNRs of all links scale in proportion to the average SNR of the $\mathcal{R}_{1}-\mathcal{D}$ link. It is easy to see that the terms $\operatorname{Pr}\left\{\alpha_{\mathrm{s}, 1} \geq \frac{g_{\mathrm{s}, 2} \alpha_{\mathrm{s}, 2}}{g_{\mathrm{s}, 1}}\right\}$ and $\operatorname{Pr}\left\{\alpha_{\mathrm{s}, 2} \geq \frac{g_{\mathrm{s}, 1} \alpha_{\mathrm{s}, 1}}{g_{\mathrm{s}, 2}}\right\}$ of (31) and (32), respectively, do not affect the diversity. Then, after analyzing the negative powers of $\bar{\gamma}$ in (31) and (32), we find that the diversity order of the proposed selection scheme is $\min \left(\min \left(\mu_{\mathrm{d}, 1} \triangleq n_{\mathrm{r}, 1} n_{\mathrm{d}}, \mu_{\mathrm{d}, 2} \triangleq n_{\mathrm{r}, 2} n_{\mathrm{d}}\right), \mu_{\mathrm{s}, 1}+\mu_{\mathrm{s}, 2} \triangleq\right.$ $\left.n_{\mathrm{s}} n_{\mathrm{r}, 1}+n_{\mathrm{s}} n_{\mathrm{r}, 2}\right)$. The following important observations can be made from the above asymptotic analysis.

Remark 4: When perfect global CSI is available at the source, i.e., when the $q$ th relay also feeds back information on instantaneous $\mathcal{R}_{q}-\mathcal{D}$ channel to the source, OR can be implemented. In such a case, the outage probability at the destination simplifies to the product of the outage probabilities of the two-hop links corresponding to the first and second relays. To illustrate this fact, we first note that the proposed transmission protocol with OR leads to the following outage probability at the destination:

$$
\begin{array}{r}
P_{\text {out }}^{\mathrm{P}}=\operatorname{Pr}\left\{\frac{e_{\mathrm{d}, 1} \alpha_{\mathrm{d}, 1} g_{\mathrm{s}, 1} \alpha_{\mathrm{s}, 1}}{e_{\mathrm{d}, 1} \alpha_{\mathrm{d}, 1}+g_{\mathrm{s}, 1} \alpha_{\mathrm{s}, 1}+1} \leq \gamma_{\mathrm{th}},\right. \\
\left.\frac{e_{\mathrm{d}, 2} \alpha_{\mathrm{d}, 2} g_{\mathrm{s}, 2} \alpha_{\mathrm{s}, 2}}{e_{\mathrm{d}, 2} \alpha_{\mathrm{d}, 2}+g_{\mathrm{s}, 2} \alpha_{\mathrm{s}, 2}+1} \leq \gamma_{\mathrm{th}}\right\}
\end{array}
$$




$$
\begin{aligned}
& =(a) \prod_{q=1}^{2} \operatorname{Pr}\left\{\frac{e_{\mathrm{d}, q} \alpha_{\mathrm{d}, q} g_{\mathrm{s}, q} \alpha_{\mathrm{s}, q}}{e_{\mathrm{d}, q} \alpha_{\mathrm{d}, q}+g_{\mathrm{s}, q} \alpha_{\mathrm{s}, q}+1} \leq \gamma_{\mathrm{th}}\right\} \\
& =\left(\left.I\right|_{g_{\mathrm{s}, 2}=0}\right)\left(\left.I I\right|_{g_{\mathrm{s}, 1}=0}\right) \\
& \approx{ }^{(b)} \prod_{q=1}^{2} \operatorname{Pr}\left\{\min \left(e_{\mathrm{d}, q} \alpha_{\mathrm{d}, q}, g_{\mathrm{s}, q} \alpha_{\mathrm{s}, q}\right) \leq \gamma_{\mathrm{th}}\right\} \\
& ={ }^{(c)} \prod_{q=1}^{2}\left(1-\operatorname{Pr}\left\{e_{\mathrm{d}, q} \alpha_{\mathrm{d}, q} \geq \gamma_{\mathrm{th}}\right\} \operatorname{Pr}\left\{g_{\mathrm{s}, q} \alpha_{\mathrm{s}, q} \geq \gamma_{\mathrm{th}}\right\}\right)
\end{aligned}
$$

where $(a)$ follows from the fact that $\operatorname{Pr}\{X, Y\}=$ $\operatorname{Pr}\{X\} \operatorname{Pr}\{Y\}$ for statistically independent random events $X$ and $Y,\left.I\right|_{g_{\mathrm{s}, 2}=0}$ and $\left.I I\right|_{g_{\mathrm{s}, 1}=0}$ denote the values of probability terms $I$ and $I I$ of (13) for $g_{\mathrm{s}, 2}=0$ and $g_{\mathrm{s}, 1}=0$, respectively, (b) is due to the application of (26) and $(c)$ is due to the facts that $\operatorname{Pr}\left\{X \leq x_{0}\right\}=1-\operatorname{Pr}\left\{X \geq x_{0}\right\}$ and $\min (X, Y) \geq x_{0}$ can be recast as the combined event $\left(X \geq x_{0}, Y \geq x_{0}\right)$. After expanding (33) and using (25), we obtain

$$
P_{\mathrm{out}}^{\mathrm{P}} \approx \prod_{q=1}^{2}\left[\frac{r_{\mathrm{d}, q} \gamma_{\mathrm{th}}^{\mu_{\mathrm{d}, q}}}{\mu_{\mathrm{d}, q} e_{\mathrm{d}, q}^{\mu_{\mathrm{d}, q}}}+\frac{r_{\mathrm{s}, q} \gamma_{\mathrm{th}, q}^{\mu_{\mathrm{s}, q}}}{\mu_{\mathrm{s}, q} g_{\mathrm{s}, q}^{\mu_{\mathrm{s}, q}}}\left(1-\frac{r_{\mathrm{d}, q} \gamma_{\mathrm{th}}^{\mu_{\mathrm{d}, q}}}{\mu_{\mathrm{d}, q} e_{\mathrm{d}, q}^{\mu_{\mathrm{d}, q}}}\right)\right]
$$

Again, assuming $e_{\mathrm{d}, 1}=\bar{\gamma}, e_{\mathrm{d}, 2}=\nu_{1} \bar{\gamma}, g_{\mathrm{s}, 1}=\nu_{2} \bar{\gamma}, g_{\mathrm{s}, 2}=\nu_{3} \bar{\gamma}$ w.l.o.g, it is straightforward to show that there are four terms that dominate the asymptotic outage probability of (34). These terms decrease with $\bar{\gamma}^{-\left(\mu_{\mathrm{d}, 1}+\mu_{\mathrm{d}, 2}\right)}, \bar{\gamma}^{-\left(\mu_{\mathrm{d}, 1}+\mu_{\mathrm{s}, 2}\right)}$, $\bar{\gamma}^{-\left(\mu_{\mathrm{s}, 1}+\mu_{\mathrm{d}, 2}\right)}$, and $\bar{\gamma}^{-\left(\mu_{\mathrm{s}, 1}+\mu_{\mathrm{s}, 2}\right)}$. Consequently, the diversity order of $\min \left(\mu_{\mathrm{d}, 1}+\mu_{\mathrm{d}, 2}, \mu_{\mathrm{d}, 1}+\mu_{\mathrm{s}, 2}, \mu_{\mathrm{s}, 1}+\mu_{\mathrm{d}, 2}, \mu_{\mathrm{s}, 1}+\mu_{\mathrm{s}, 2}\right)$ is achieved, which is also the maximum diversity order of the system.

Remark 5: We have shown that the diversity order of the PRS scheme for the considered multiantenna setup is $\min \left(\min \left(n_{\mathrm{r}, 1} n_{\mathrm{d}}, n_{\mathrm{r}, 2} n_{\mathrm{d}}\right), n_{\mathrm{s}} n_{\mathrm{r}, 1}+n_{\mathrm{s}} n_{\mathrm{r}, 2}\right)$, which, in general, is of course lower than the diversity order $\min \left(n_{\mathrm{d}} n_{\mathrm{r}, 1}+\right.$ $\left.n_{\mathrm{d}} n_{\mathrm{r}, 2}, n_{\mathrm{d}} n_{\mathrm{r}, 1}+n_{\mathrm{s}} n_{\mathrm{r}, 2}, n_{\mathrm{s}} n_{\mathrm{r}, 1}+n_{\mathrm{d}} n_{\mathrm{r}, 2}, n_{\mathrm{s}} n_{\mathrm{r}, 1}+n_{\mathrm{s}} n_{\mathrm{r}, 2}\right)$ of OR scheme. This means that for the general antenna configuration, the PRS method does not achieve the maximum diversity order. This is due to the fact that the selection is based on the assumption that the source does not have any CSI of the channels from relays to the destination. However, when the condition $C: \min \left(n_{\mathrm{r}, 1} n_{\mathrm{d}}, n_{\mathrm{r}, 2} n_{\mathrm{d}}\right) \geq n_{\mathrm{s}} n_{\mathrm{r}, 1}+n_{\mathrm{s}} n_{\mathrm{r}, 2}$ is satisfied, the PRS scheme gives the same diversity order as that of OR scheme. For $n_{\mathrm{r}, 1}=n_{\mathrm{r}, 2}$, this implies that $n_{\mathrm{d}} \geq 2 n_{\mathrm{s}}$. Such antenna configurations may be typical in an uplink communication system where the destination (base station) has more resources and processing capability than a source (mobile station).

Remark 6: It is important to emphasize how our results can be valuable for practical systems. Note that PRS incurs no loss in diversity if the antenna configurations meet the condition $C$. This condition can be met with practically realizable antenna configurations. To illustrate further, consider an example $n_{\mathrm{s}}=$ $n_{\mathrm{r}, 1}=n_{\mathrm{r}, 2}=2$ and $n_{\mathrm{d}}=4$. According to our results, PRS and OR achieve the same diversity order of 8 . Noting that PRS does not require feedback (any type) of CSI of $\mathcal{R}_{q}-\mathcal{D}$ channel whereas the OR relies on this feedback, our results on diversity confirm that the feedback information sent from the relays to the source may not be useful to improve the diversity gain for antenna configurations that meet the condition $C$.

\section{A. Diversity Order for $n_{\mathrm{d}}=n_{\mathrm{r}, 1}=n_{\mathrm{r}, 2}=n_{\mathrm{s}}=1$}

The diversity order for this case can be determined easily from the asymptotic analysis of (23). Note that, for small $x$, we know that $K_{1}(x) \approx \frac{1}{x}$ and $\mathrm{e}^{-x} \approx 1-x$. After recalling the definitions of $\tilde{\alpha}$ and $\tilde{\beta}$, we can notice that these parameters become small for large values of $\left\{g_{\mathrm{s}, q}\right\}_{q=1}^{2}$ and $\left\{e_{\mathrm{d}, q}\right\}_{q=1}^{2}$, respectively. Consequently, after applying the asymptotic approximations for the functions $K_{1}(x)$ and $\mathrm{e}^{-x}$, the asymptotic expression for (23) can be given as

$$
\tilde{P}_{\text {out }} \approx \gamma_{\mathrm{th}} \tilde{\beta} \pm \mathcal{O}\left((2 \tilde{\alpha}+\tilde{\beta}) \gamma_{\mathrm{th}}\right) \pm 2 \mathcal{O}\left((\tilde{\alpha}+\tilde{\beta}) \gamma_{\mathrm{th}}\right)
$$

where $\mathcal{O}(y)$ stands for higher powers of $y$. From (35), it can be seen that the diversity order of the proposed selection method for $n_{\mathrm{d}}=n_{\mathrm{r}, 1}=n_{\mathrm{r}, 2}=n_{\mathrm{s}}=1$ remains 1 . This result is in agreement with that of [18] where all nodes are considered to be equipped with only one antenna.

\section{NumERICAL RESUlTS AND DisCUSSIONS}

In this section, we first provide Monte Carlo simulations to assess the accuracy of the closed-form expressions for the outage probabilities (13) which is calculated with the help of (17) and (18)-(20). We also ascertain the analytically derived diversity gains for different antenna configurations. In all examples, we take $\sigma_{\mathrm{n}_{\mathrm{d}}}^{2}=\sigma_{\mathrm{n}_{\mathrm{r}}}^{2}=\sigma_{n}^{2}, P_{\mathrm{S}}=P_{\mathrm{R}, q}=P, \forall q$ and vary the value of $P$ while keeping $\sigma_{n}^{2}=1$. The average SNR is defined as $\mathcal{S N} \mathcal{R}=\frac{P}{\sigma_{n}^{2}}$. For all results, we take $\gamma_{\mathrm{th}}=3 \mathrm{~dB}(\mathrm{ex}-$ cept in Fig. 3 and 5 where $\gamma_{\mathrm{th}}$ varies), $c_{\mathrm{d}, 1}=c_{\mathrm{d}, 2}=0.98$ and $c_{\mathrm{s}, 1}=c_{\mathrm{s}, 2}=0.95$. Note that this is purely by way of example, and any other values could just have been used. The outage probability results are obtained using Monte Carlo simulation runs during which the elements of MIMO channels $\left\{\mathbf{H}_{\mathrm{s}, q}\right\}_{q=1}^{2}$ and $\left\{\mathbf{H}_{\mathrm{d}, q}\right\}_{q=1}^{2}$ change independently and randomly. The entries of all MIMO channels are taken to be ZMCSCG random variables with unit variance. The coefficients $d_{i_{q}, m_{q}}$ and $d_{j_{q}, n_{q}}$ for different antenna configurations are directly taken from the tables of [29].

The theoretical and simulated outage probabilities as a function of $\mathcal{S} \mathcal{N} \mathcal{R}$ are shown in Fig. 2 for different values of $n_{\mathrm{d}}$. In this figure, we take $n_{\mathrm{r}, 1}=n_{\mathrm{r}, 2}=n_{\mathrm{s}}=2$. In Fig. 3, the outage probability is displayed as a function of the threshold value $\gamma_{\mathrm{th}}$ of the destination SNR for different $n_{\mathrm{d}}$ while keeping $n_{\mathrm{r}, 1}=n_{\mathrm{r}, 2}=n_{\mathrm{s}}=2$ and $\mathcal{S} \mathcal{N} \mathcal{R}=5 \mathrm{~dB}$. In Fig. 4, analytical and simulated outage probabilities are compared for different values of $n_{\mathrm{s}}$ whereas $n_{\mathrm{r}, 1}=n_{\mathrm{r}, 2}=2$, and $n_{\mathrm{d}}=6$ are assumed. Again, the outage probabilities are displayed as a function of $\mathcal{S N} \mathcal{R}$ in this figure. In Fig. 5, theoretical and simulation results for outage probability have been plotted as a function of $\gamma_{\mathrm{th}}$ for different $n_{\mathrm{s}}$. We take $n_{\mathrm{r}, 1}=n_{\mathrm{r}, 2}=2, n_{\mathrm{d}}=6$, and $\mathcal{S N} \mathcal{R}=5 \mathrm{~dB}$ in Fig. 5. As expected, in Fig. 2 and Fig. 4, the outage probability decreases for all cases when $S \mathcal{N} \mathcal{R}$ increases whereas in Fig. 3 and Fig. 5, the outage probability increases for increasing QoS requirements. Figs. 2-5 also demonstrate that there is a fine agreement between the theoretical and simulation results which verifies the correctness of our proposed outage probability analysis.

Note that in Figs. 2 and 4, we have also plotted the curves proportional to $\mathcal{S} \mathcal{N} \mathcal{R}^{-\mathrm{d}_{0}}$ where $\mathrm{d}_{0}$ is an integer value that denotes the diversity gain. The fine matching between these curves 


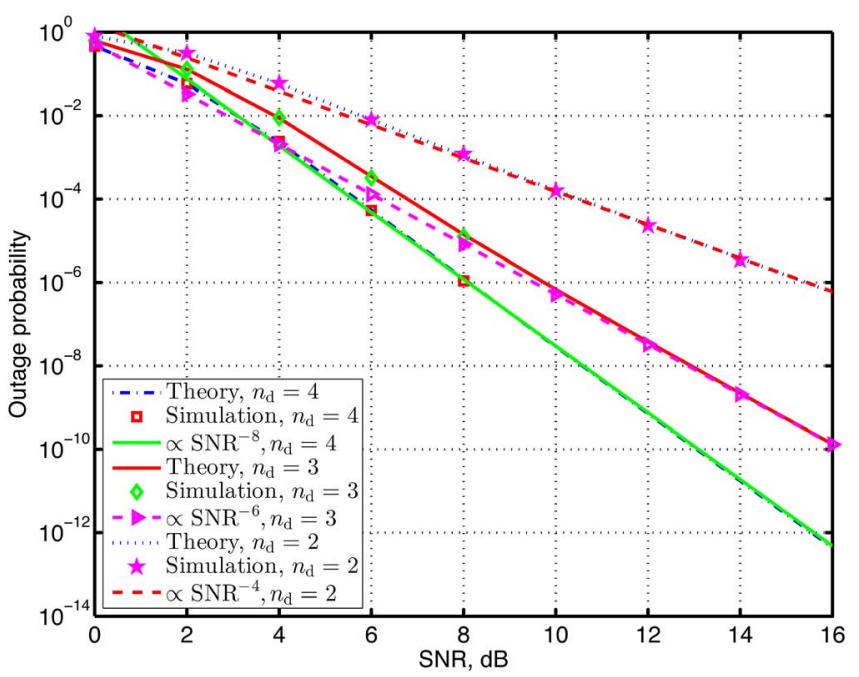

Fig. 2. Analytical and simulated outage probabilities as a function of average $\operatorname{SNR}\left(n_{\mathrm{s}}=n_{\mathrm{r}, 1}=n_{\mathrm{r}, 2}=2\right)$.

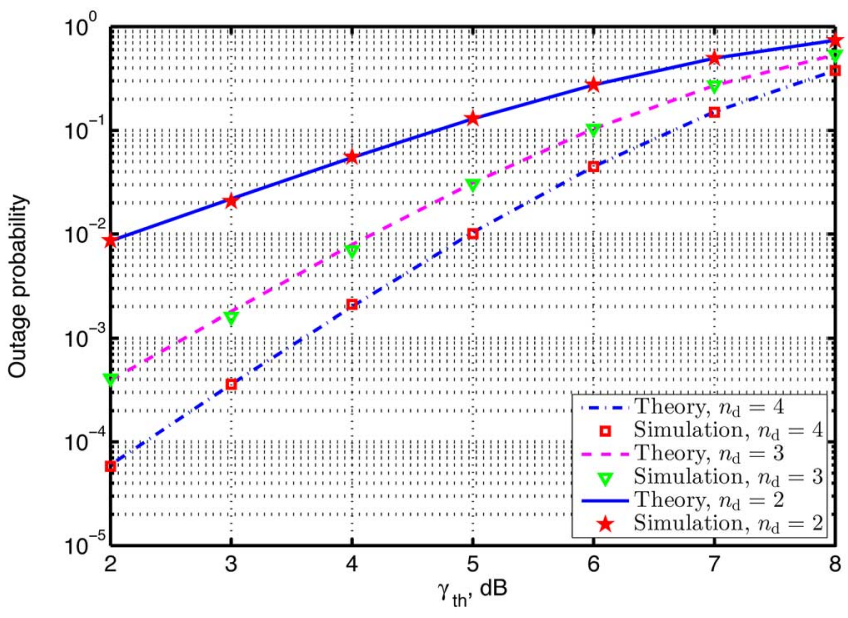

Fig. 3. Analytical and simulated outage probabilities as a function of $\gamma_{\mathrm{th}}$ $\left(n_{\mathrm{s}}=n_{\mathrm{r}, 1}=n_{\mathrm{r}, 2}=2\right)$.

and the theoretically obtained outage probability curves at high values of $\mathcal{S} \mathcal{N} \mathcal{R}$ confirms that the diversity gain results are in accordance with our theoretically determined diversity order of $\min \left(\min \left(n_{\mathrm{r}, 1} n_{\mathrm{d}}, n_{\mathrm{r}, 2} n_{\mathrm{d}}\right), n_{\mathrm{r}, 1} n_{\mathrm{s}}+n_{\mathrm{r}, 2} n_{\mathrm{s}}\right)$.

\section{CONCLUSION}

We have investigated PRS method that performs physical layer routing for a multiantenna cooperative network and analyzed its outage performance. The proposed analysis is based upon a setup that employs transmit and receive beamforming techniques respectively at the source and destination, and receive plus transmit beamforming at the relay nodes. The exact closed-form expression for the end-to-end outage probability has been obtained for the PRS based routing scheme where all the nodes only have their respective local CSI. The asymptotic analysis of the outage probability is presented and expressions for diversity gain have been derived. Despite the fact that the PRS scheme does not assume instantaneous global CSI, it provides the same diversity order as the OR scheme for the case where $\min \left(n_{\mathrm{r}, 1} n_{\mathrm{d}}, n_{\mathrm{r}, 2} n_{\mathrm{d}}\right) \geq n_{\mathrm{s}} n_{\mathrm{r}, 1}+n_{\mathrm{s}} n_{\mathrm{r}, 2}$. Consequently,

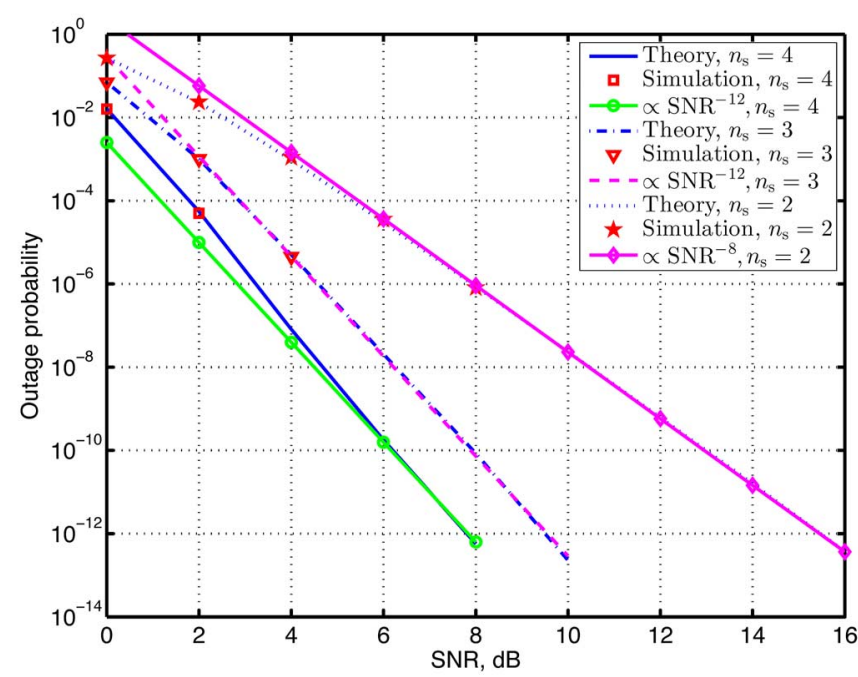

Fig. 4. Comparison of analytical and simulated outage probabilities as a function of average $\operatorname{SNR}\left(n_{\mathrm{d}}=6, n_{\mathrm{r}, 1}=n_{\mathrm{r}, 2}=2\right)$.

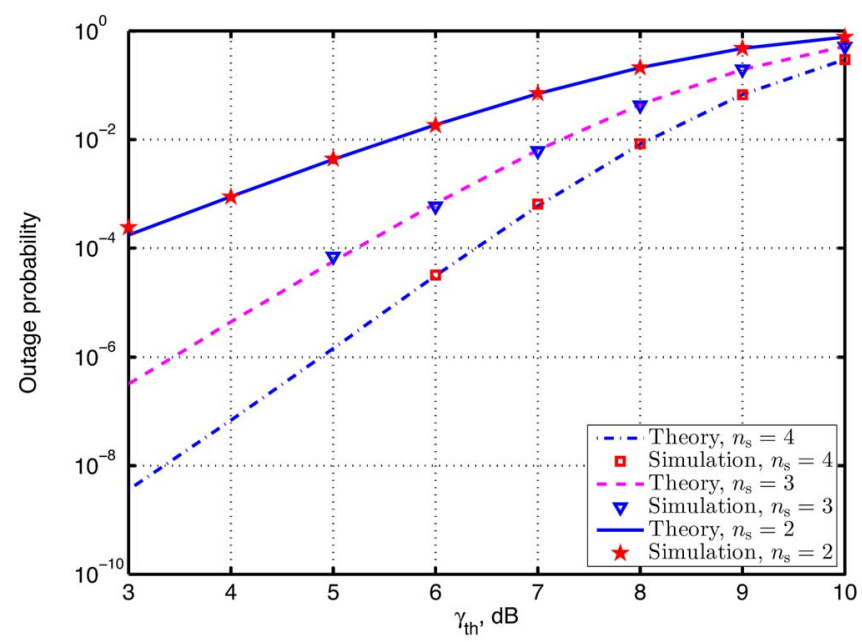

Fig. 5. Comparison of analytical and simulated outage probabilities as a function of $\gamma_{\mathrm{th}}\left(n_{\mathrm{d}}=6, n_{\mathrm{r}, 1}=n_{\mathrm{r}, 2}=2\right)$.

for the antenna configurations that meet this condition, there is no need to feed back any CSI of relay-destination channels to the source for achieving the full diversity.

\section{APPENDIX A}

\section{DERIVATION OF (17)}

In (15), $\tilde{\alpha}_{\mathrm{s}, 2}=d_{\mathrm{c}} \alpha_{\mathrm{s}, 2}$ whose PDF is simply obtained from (8) as

$$
\begin{aligned}
f_{\tilde{\alpha}_{s, 2}}(y) & =\frac{1}{d_{\mathrm{c}}} f_{\alpha_{\mathrm{s}, 2}}\left(\frac{y}{d_{\mathrm{c}}}\right) \\
& =\sum_{i_{2}=1}^{a_{1,2}} \sum_{m_{2}=b_{1,2}}^{b_{2,2}} \frac{d_{i_{2}, m_{2}}}{m_{2} !}\left(\frac{i_{2}}{d_{\mathrm{c}}}\right)^{m_{2}+1} y^{m_{2}} \mathrm{e}^{-\frac{i_{2} y}{d_{\mathrm{c}}}} .
\end{aligned}
$$

Applying the PDF of $\alpha_{\mathrm{d}, 1}$ from (8) into (15), and using the following expression [30, eq. (3.381.1)]:

$$
\int_{0}^{u} x^{v-1} \mathrm{e}^{-\mu x} d x=\mu^{-v} \gamma_{\mathrm{L}}(v, \mu u), v>0
$$


where $\gamma_{\mathrm{L}}(\cdot, \cdot)$ is the lower incomplete Gamma function, we can write (15) as

$$
\begin{aligned}
& {[I]_{z=0}^{\frac{\gamma_{\mathrm{th}}}{e_{\mathrm{d}, 1}}}=\operatorname{Pr}\left\{\alpha_{\mathrm{s}, 1} \geq \tilde{\alpha}_{\mathrm{s}, 2}\right\} \sum_{j_{1}=1}^{a_{2,1}} \sum_{n_{1}=c_{1,1}}^{c_{2,1}} \frac{d_{j_{1}, n_{1}}}{n_{1} !}} \\
& \times \gamma_{\mathrm{L}}\left(n_{1}+1, \frac{j_{1} \gamma_{\mathrm{th}}}{e_{\mathrm{d}, 1}}\right) .
\end{aligned}
$$

In the following, we complete the remaining task of deriving the term $\operatorname{Pr}\left\{\alpha_{\mathrm{s}, 1} \geq \tilde{\alpha}_{\mathrm{s}, 2}\right\}$. Noting that

$$
\operatorname{Pr}\left\{\alpha_{\mathrm{s}, 1} \geq \tilde{\alpha}_{\mathrm{s}, 2}\right\}=1-\operatorname{Pr}\left\{W \triangleq \frac{\alpha_{\mathrm{s}, 1}}{\tilde{\alpha}_{\mathrm{s}, 2}} \leq 1\right\}
$$

where $W$ is the ratio of two independent random variables $\alpha_{\mathrm{s}, 1}$ and $\tilde{\alpha}_{\mathrm{s}, 2}$, the PDF of $W$ can be given by

$$
f_{W}(w)=\int_{0}^{\infty} y f_{\alpha_{s, 1}}(w y) f_{\tilde{\alpha}_{s, 2}}(y) d y .
$$

Substituting (8) and (36) into (40), and applying some straightforward derivations, we can obtain

$$
\begin{aligned}
f_{W}(w) & =\sum_{i_{1}=1}^{a_{1,1}} \sum_{m_{1}=b_{1,1}}^{b_{2,1}} \sum_{i_{2}=1}^{a_{1,2}} \sum_{m_{2}=b_{1,2}}^{b_{2,2}} \frac{d_{i_{1}, m_{1}} d_{i_{2}, m_{2}} i_{1}^{m_{1}+1}}{m_{1} ! m_{2} ! d_{\mathrm{c}}^{m_{2}+1}} \\
& \times i_{2}^{m_{2}+1} \Gamma\left(m_{1}+m_{2}+2\right) \frac{w^{m_{1}}}{\left(i_{1} w+\frac{i_{2}}{d_{c}}\right)^{m_{1}+m_{2}+2}}
\end{aligned}
$$

where we have used [30, eq. (3.381.4)]. With the help of (41), we can write (39) as

$$
\begin{aligned}
\operatorname{Pr}\left\{\alpha_{\mathrm{s}, 1} \geq \tilde{\alpha}_{\mathrm{s}, 2}\right\}= & 1-\int_{0}^{1} f_{W}(w) d w \\
= & 1-\sum_{i_{1}=1}^{a_{1,1}} \sum_{m_{1}=b_{1,1}}^{b_{2,1}} \sum_{i_{2}=1}^{a_{2,2}} \sum_{m_{2}=b_{1,2}}^{b_{2,2}} \frac{d_{i_{1}, m_{1}}}{m_{1} ! m_{2} !} \\
& \times d_{i_{2}, m_{2}} \Gamma\left(m_{1}+m_{2}+2\right) \\
& \times \frac{d_{\mathrm{c}}^{m_{1}+1}}{i_{2}^{m_{1}+m_{2}+2}} i_{1}^{m_{1}+1} i_{2}^{m_{2}+1} \\
& \times \int_{0}^{1} \frac{w^{m_{1}}}{\left[1+\frac{i_{1} d_{c}}{i_{2}} w\right]^{m_{1}+m_{2}+2}} d w
\end{aligned}
$$

Applying [30, eq. (3.194.1)] in (42), we get (42) in terms of the Gauss hypergeometric function ${ }_{2} F_{1}()$ as

$$
\begin{aligned}
\operatorname{Pr} & \left\{\alpha_{\mathrm{s}, 1} \geq \tilde{\alpha}_{\mathrm{s}, 2}\right\} \\
= & 1-\sum_{i_{1}=1}^{a_{1,1}} \sum_{m_{1}=b_{1,1}}^{b_{2,1}} \sum_{i_{2}=1}^{a_{1,2}} \sum_{m_{2}=b_{1,2}}^{b_{2,2}} \frac{d_{i_{1}, m_{1}}}{m_{1} ! m_{2} !} \\
& \times d_{i_{2}, m_{2}} \Gamma\left(m_{1}+m_{2}+2\right) \frac{i_{1}^{m_{1}+1} d_{\mathrm{c}}^{m_{1}+1}}{i_{2}^{m_{1}+1}\left(m_{1}+1\right)} \\
& \times{ }_{2} F_{1}\left(m_{1}+m_{2}+2, m_{1}+1 ; m_{1}+2 ;-\frac{i_{1} d_{\mathrm{c}}}{i_{2}}\right) .
\end{aligned}
$$

After substituting (43) into (38), we get the desired expression (17).

\section{APPENDIX B \\ DERIVATION OF (18)}

It is evident that the probability term in (16) is zero for $y>$ $\frac{\gamma_{\mathrm{th}}\left(e_{\mathrm{d}, 1} z+1\right)}{g_{\mathrm{s}, 1}\left(e_{\mathrm{d}, 1} z-\gamma_{\mathrm{th}}\right)}$ which leads to $y_{u}(z)=\frac{\gamma_{\mathrm{th}}\left(e_{\mathrm{d}, 1} z+1\right)}{g_{\mathrm{s}, 1}\left(e_{\mathrm{d}, 1} z-\gamma_{\mathrm{th}}\right)}$. Noting that $\operatorname{Pr}\left\{X \leq x_{1}, X \geq x_{2}\right\}=\operatorname{Pr}\left\{X \leq x_{1}\right\}-\operatorname{Pr}\left\{X \leq x_{2}\right\}$ for a positive random variable $X$ and the positive constants $x_{1}$ and $x_{2}$ with $x_{1} \geq x_{2},(16)$ can be given by

$$
\begin{aligned}
& {[I]_{z=\frac{\gamma_{\mathrm{th}}}{e_{\mathrm{d}, 1}}}^{\infty}=\int_{\frac{\gamma_{\mathrm{th}}}{\epsilon_{\mathrm{d}, 1}}}^{\infty} \operatorname{Pr}\left\{\alpha_{\mathrm{s}, 1} \leq \frac{\gamma_{\mathrm{th}}\left(e_{\mathrm{d}, 1} z+1\right)}{g_{\mathrm{s}, 1}\left(e_{\mathrm{d}, 1} z-\gamma_{\mathrm{th}}\right)}\right\}} \\
& \frac{\gamma_{\mathrm{th}}\left(e_{\mathrm{d}, 1} z+1\right)}{g_{\mathrm{s}, 1}\left(e_{\mathrm{d}, 1} z-\gamma_{\mathrm{th}}\right)} \\
& \times \underbrace{\int_{0} f_{\tilde{\alpha}_{\mathrm{s}, 2}}(y) d y}_{I_{1}} f_{\alpha_{\mathrm{d}, 1}}(z) d z \\
& -\int_{\frac{\gamma_{\mathrm{th}}}{e_{\mathrm{d}, 1}}}^{\infty} \underbrace{\int_{0}^{\frac{\gamma_{\mathrm{th}}\left(e_{\mathrm{d}, 1} z+1\right)}{g_{\mathrm{s}, 1}\left(e_{\mathrm{d}, 1}{ }^{\left.z-\gamma_{\mathrm{th}}\right)}\right.}} \operatorname{Pr}\left\{\alpha_{\mathrm{s}, 1} \leq y\right\} f_{\tilde{\alpha}_{\mathrm{s}, 2}}(y) d y}_{I_{2}} \\
& \times f_{\alpha_{\mathrm{d}, 1}}(z) d z .
\end{aligned}
$$

Using the PDF of $\alpha_{\mathrm{s}, 1}$ from (8) and applying (37), we can write

$$
\begin{array}{r}
\operatorname{Pr}\left\{\alpha_{\mathrm{s}, 1} \leq \frac{\gamma_{\mathrm{th}}\left(e_{\mathrm{d}, 1} z+1\right)}{g_{\mathrm{s}, 1}\left(e_{\mathrm{d}, 1} z-\gamma_{\mathrm{th}}\right)}\right\}=\sum_{i_{1}=1}^{a_{1,1}} \sum_{m_{1}=b_{1,1}}^{b_{2,1}} \frac{d_{i_{1}, m_{1}}}{m_{1} !} \\
\times \gamma_{\mathrm{L}}\left(m_{1}+1, \frac{i_{1} \gamma_{\mathrm{th}}\left(e_{\mathrm{d}, 1} z+1\right)}{g_{\mathrm{s}, 1}\left(e_{\mathrm{d}, 1} z-\gamma_{\mathrm{th}}\right)}\right) .
\end{array}
$$

Using (36) and (37), and after some derivations, we obtain

$$
I_{1}=\sum_{i_{2}=1}^{a_{1,2}} \sum_{m_{2}=b_{1,2}}^{b_{2,2}} \frac{d_{i_{2}, m_{2}}}{m_{2} !} \gamma_{\mathrm{L}}\left(m_{2}+1, \frac{i_{2} \gamma_{\mathrm{th}}\left(e_{\mathrm{d}, 1} z+1\right)}{d_{\mathrm{c},} g_{\mathrm{s}, 1}\left(e_{\mathrm{d}, 1} z-\gamma_{\mathrm{th}}\right)}\right) \text {. }
$$

In order to calculate $I_{2}$, we first derive $\operatorname{Pr}\left\{\alpha_{\mathrm{s}, 1} \leq y\right\}$ using the PDF of $\alpha_{s, 1}$ in (8) and apply (37). Thus, we have

$$
\begin{aligned}
\operatorname{Pr}\left\{\alpha_{\mathrm{s}, 1} \leq y\right\} & =\sum_{i_{1}=1}^{a_{1,1}} \sum_{m_{1}=b_{1,1}}^{b_{2,1}} \frac{d_{i_{1}, m_{1}}}{m_{1} !} \gamma_{\mathrm{L}}\left(m_{1}+1, i_{1} y\right) \\
& =1-\sum_{i_{1}=1}^{a_{1,1}} \sum_{m_{1}=b_{1,1}}^{b_{2,1}} \sum_{p=0}^{m_{1}} \frac{d_{i_{1}, m_{1}}\left(i_{1} y\right)^{p}}{p !} \mathrm{e}^{-i_{1} y}
\end{aligned}
$$

where the second equality is obtained after utilizing the series expansion for $\gamma_{\mathrm{L}}(\cdot, \cdot)$ (see [30, eq. (8.352.1)]) and using (9). With the help of (47), we can express $I_{2}$ as

$$
\begin{aligned}
I_{2}= & \int_{0}^{\frac{\gamma_{\mathrm{th}}\left(e_{\mathrm{d}, 1} z+1\right)}{g_{\mathrm{s}, 1}\left(e_{\mathrm{d}, 1} z-\gamma_{\mathrm{th}}\right)}} f_{\tilde{\alpha}_{\mathrm{s}, 2}}(y) d y-\sum_{i_{1}=1}^{a_{1,1}} \sum_{m_{1}=b_{1,1}}^{b_{2,1}} \sum_{p=0}^{m_{1}} \frac{d_{i_{1}, m_{1}}\left(i_{1}\right)^{p}}{p !} \\
& \times \int_{0}^{\frac{\gamma_{\mathrm{th}}\left(e_{\mathrm{d}, 1} z+1\right)}{g_{\mathrm{s}, 1}\left(e_{\left.\mathrm{d}, 1, \gamma_{\mathrm{th}}\right)}\right.}} y^{p} \mathrm{e}^{-i_{1} y} f_{\tilde{\alpha}_{\mathrm{s}, 2}}(y) d y .
\end{aligned}
$$


Substituting (36) into (48), and using (9), we obtain

$$
\begin{aligned}
I_{2}= & \sum_{i_{2}=1}^{a_{1,2}} \sum_{m_{2}=b_{1,2}}^{b_{2,2}} \frac{d_{i_{2}, m_{2}}}{m_{2} !} \gamma_{\mathrm{L}}\left(m_{2}+1, \frac{i_{2} \gamma_{\mathrm{th}}\left(e_{\mathrm{d}, 1} z+1\right)}{d_{\mathrm{c}} g_{\mathrm{s}, 1}\left(e_{\mathrm{d}, 1} z-\gamma_{\mathrm{th}}\right)}\right) \\
& -\sum_{i_{2}=1}^{a_{2,2}} \sum_{m_{2}=b_{1,2}}^{b_{2,2}} \sum_{i_{1}=1}^{a_{1,1}} \sum_{m_{1}=b_{1,1}}^{b_{2,1}} \sum_{p=0}^{m_{1}} \frac{d_{i_{2}, m_{2}} d_{i_{1}, m_{1}}\left(i_{1}\right)^{p}}{m_{2} ! p !} \\
& \times\left(\frac{i_{2}}{d_{\mathrm{c}}}\right)^{m_{2}+1}\left(i_{1}+\frac{i_{2}}{d_{\mathrm{c}}}\right)^{-\left(m_{2}+p+1\right)} \\
& \times \gamma_{\mathrm{L}}\left(m_{2}+p+1,\left(i_{1}+\frac{i_{2}}{d_{\mathrm{c}}}\right) \frac{\gamma_{\mathrm{th}}\left(e_{\mathrm{d}, 1} z+1\right)}{g_{\mathrm{s}, 1}\left(e_{\mathrm{d}, 1} z-\gamma_{\mathrm{th}}\right)}\right) .
\end{aligned}
$$

After computing the inner integrals $I_{1}$ and $I_{2}$ of (44) and having (45), we can proceed to compute the outer integral of (44) with respect to the variable $z$. The computation of the latter integral is rather involved. Using the alternative form of (45) (obtained after [30, eq. (8.352.1)]), (46), (49), (9), and substituting $\bar{z}=$ $e_{\mathrm{d}, 1} z-\gamma_{\mathrm{th}},(44)$ can be given by (18).

\section{APPENDIX C}

DERIVATION OF (19)

Applying the series expansion of lower incomplete Gamma function [30, eq. (8.352.1) ] in (18), its first integral can be expressed as

$$
\begin{aligned}
I_{3}= & m_{2} ! \int_{0}^{\infty} \bar{z}^{-q}\left(\bar{z}+\gamma_{\mathrm{th}}+1\right)^{q} \mathrm{e}^{-\hat{d}_{2} \bar{z}^{-1}} f_{\alpha_{\mathrm{d}, 1}}\left(\frac{\bar{z}+\gamma_{\mathrm{th}}}{e_{\mathrm{d}, 1}}\right) \\
& \times \frac{1}{e_{\mathrm{d}, 1}} d \bar{z}-m_{2} ! \mathrm{e}^{-d_{1}} \sum_{u=0}^{m_{2}} \frac{d_{1}^{u}}{u !} \int_{0}^{\infty}\left(\bar{z}+\gamma_{\mathrm{th}}+1\right)^{q+u} \\
& \times \bar{z}^{-u-q} \mathrm{e}^{-\tilde{d}_{2} \bar{z}^{-1}} f_{\alpha_{\mathrm{d}, 1}}\left(\frac{\bar{z}+\gamma_{\mathrm{th}}}{e_{\mathrm{d}, 1}}\right) \frac{1}{e_{\mathrm{d}, 1}} d \bar{z}
\end{aligned}
$$

where for brevity we have used the following notations:

$$
\begin{aligned}
& d_{1} \triangleq \frac{i_{2} \gamma_{\mathrm{th}}}{g_{\mathrm{s}, 1} d_{\mathrm{c}}}, \quad \hat{d}_{2} \triangleq \frac{i_{1} \gamma_{\mathrm{th}}\left(\gamma_{\mathrm{th}}+1\right)}{g_{\mathrm{s}, 1}} \\
& \tilde{d}_{2} \triangleq\left(i_{1}+\frac{i_{2}}{d_{\mathrm{c}}}\right) \frac{\gamma_{\mathrm{th}}\left(\gamma_{\mathrm{th}}+1\right)}{g_{\mathrm{s}, 1}} .
\end{aligned}
$$

Using the PDF of $\alpha_{\mathrm{d}, 1}$ from (8), we have

$$
\begin{aligned}
f_{\alpha_{\mathrm{d}, 1}}\left(\frac{\bar{z}+\gamma_{\mathrm{th}}}{e_{\mathrm{d}, 1}}\right) \frac{1}{e_{\mathrm{d}, 1}}=\sum_{j_{1}=1}^{a_{2,1}} \sum_{n_{1}=c_{1,1}}^{c_{2,1}} \frac{d_{j_{1}, n_{1}} j_{1}^{n_{1}+1}}{n_{1} ! e_{\mathrm{d}, 1}^{n_{1}+1}} \mathrm{e}^{-\frac{j_{1} \gamma_{\mathrm{th}}}{e_{\mathrm{d}, 1}}} \\
\times\left(\bar{z}+\gamma_{\mathrm{th}}\right)^{n_{1}} \mathrm{e}^{-j_{1} \bar{z} e_{\mathrm{d}, 1}^{-1}}
\end{aligned}
$$

Applying (52) and using the binomial expansion [30, eq. (1.111)], the first integral of (50) yields

$$
\begin{aligned}
& A_{1}=m_{2} ! \sum_{j_{1}=1}^{a_{2,1}} \sum_{n_{1}=c_{1,1}}^{c_{2,1}} \frac{d_{j_{1}, n_{1}} j_{1}^{n_{1}+1}}{n_{1} ! e_{\mathrm{d}, 1}^{n_{1}+1}} \mathrm{e}^{-j_{1} \bar{z} e_{\mathrm{d}, 1}^{-1}} \sum_{v=0}^{n_{1}}\left(\begin{array}{c}
n_{1} \\
v
\end{array}\right) \gamma_{\mathrm{th}}^{n_{1}-v} \\
& \times \sum_{w=0}^{q}\left(\begin{array}{c}
q \\
w
\end{array}\right)\left(\gamma_{\mathrm{th}}+1\right)^{q-w} \int_{0}^{\infty} \bar{z}^{v+w-q} \mathrm{e}^{-\hat{d}_{2} \bar{z}^{-1}-j_{1} \bar{z} e_{\mathrm{d}, 1}^{-1}} d \bar{z}
\end{aligned}
$$

where the integral can be evaluated as [30, eq. (3.471.9)]

$$
\begin{aligned}
& \int_{0}^{\infty} \bar{z}^{v+w-q} \mathrm{e}^{-\hat{d}_{2} \bar{z}^{-1}-j_{1} \bar{z} e_{\mathrm{d}, 1}^{-1}} d \bar{z}=2\left(\frac{\hat{d}_{2} e_{\mathrm{d}, 1}}{j_{1}}\right)^{\frac{v+w-q+1}{2}} \\
& \times K_{v+w-q+1}\left(2 \sqrt{\frac{j_{1} \hat{d}_{2}}{e_{\mathrm{d}, 1}}}\right) .
\end{aligned}
$$

Using (52), we get the second integral of (50) as

$$
\begin{aligned}
& B_{1}=m_{2} ! \mathrm{e}^{-d_{1}} \sum_{u=0}^{m_{2}} \frac{d_{1}^{u}}{u !} \sum_{j_{1}=1}^{a_{2,1}} \sum_{n_{1}=c_{1,1}}^{c_{2,1}} \frac{d_{j_{1}, n_{1}} j_{1}^{n_{1}+1}}{n_{1} ! e_{\mathrm{d}, 1}^{n+1}} \mathrm{e}^{-\frac{j_{1} \gamma_{\mathrm{th}}}{e_{\mathrm{d}, 1}}} \\
& \times \underbrace{\int_{0}^{\infty} \bar{z}^{-u-q}\left(\bar{z}+\gamma_{\mathrm{th}}+1\right)^{u+q}\left(\bar{z}+\gamma_{\mathrm{th}}\right)^{n_{1}} \mathrm{e}^{-\tilde{d}_{2} \bar{z}^{-1}-j_{1} \bar{z} e_{\mathrm{d}, 1}^{-1}} d \bar{z}}_{I_{C}} .
\end{aligned}
$$

Again applying the binomial expansion [30, eq. (1.111)] and the result (54), we have

$$
\begin{aligned}
I_{C} & =2 \sum_{a=0}^{n_{1}} \sum_{b=0}^{u+q}\left(\begin{array}{c}
n_{1} \\
a
\end{array}\right)\left(\begin{array}{c}
u+q \\
b
\end{array}\right) \gamma_{\mathrm{th}}^{n_{1}-a}\left(1+\gamma_{\mathrm{th}}\right)^{u+q-b} \\
& \times\left(\frac{\tilde{d}_{2} e_{\mathrm{d}, 1}}{j_{1}}\right)^{\frac{a+b-u-q+1}{2}} K_{a+b-u-q+1}\left(2 \sqrt{\frac{j_{1} \tilde{d}_{2}}{e_{\mathrm{d}, 1}}}\right) .
\end{aligned}
$$

This completes the derivation of (19).

\section{APPENDIX D}

\section{DERIVATION OF (20)}

Using [30, eq. (8.352.3)], applying (52) and after some derivations, we can write the second integral of (18) as

$$
\begin{gathered}
I_{4}=\left(m_{2}+p\right) ! \sum_{j_{1}=1}^{a_{2,1}} \sum_{n_{1}=c_{1,1}}^{c_{2,1}} \frac{d_{j_{1}, n_{1}} j_{1}^{n_{1}+1}}{n_{1} ! e_{\mathrm{d}, 1}^{n_{1}+1}} \mathrm{e}^{-\frac{j_{1} \gamma_{\mathrm{th}}}{e_{\mathrm{d}, 1}}} \\
\times\left\{\int_{0}^{\infty}\left(\bar{z}+\gamma_{\mathrm{th}}\right)^{n_{1}} \mathrm{e}^{-j_{1} \bar{z} e_{\mathrm{d}, 1}^{-1}} d \bar{z}-\mathrm{e}^{-c_{1}} \sum_{r=0}^{m_{2}+p} \frac{c_{1}^{r}}{r !}\right. \\
\times \int_{0}^{\infty} \bar{z}^{-r}\left(\bar{z}+\gamma_{\mathrm{th}}\right)^{n_{1}}\left(\bar{z}+\gamma_{\mathrm{th}}+1\right)^{r} \\
\left.\times \mathrm{e}^{-c_{1}\left(\gamma_{\mathrm{th}}+1\right) \bar{z}^{-1}-j_{1} \bar{z} e_{\mathrm{d}, 1}^{-1} d \bar{z}}\right\}
\end{gathered}
$$

where $c_{1} \triangleq\left(i_{1}+\frac{i_{2}}{d_{\mathrm{c}}}\right) \frac{\gamma_{\mathrm{th}}}{g_{\mathrm{s}, 1}}$. Applying [30, eq. (3.382.4)], we get the first integral of (57)

$$
I_{5}=\left(\frac{j_{1}}{e_{\mathrm{d}, 1}}\right)^{-\left(n_{1}+1\right)} \mathrm{e}^{\frac{\gamma_{\mathrm{th}} j_{1}}{e_{\mathrm{d}, 1}}} \Gamma\left(n_{1}+1, \frac{\gamma_{\mathrm{th}, j_{1}}}{e_{\mathrm{d}, 1}}\right)
$$


Using the binomial expansion (eq. (1.111) of [30]) and after some steps, we get the second integral of (57) as

$$
\begin{aligned}
I_{6}=\sum_{s=0}^{n_{1}}\left(\begin{array}{c}
n_{1} \\
s
\end{array}\right) & \gamma_{\mathrm{th}}^{n_{1}-s} \sum_{t=0}^{r}\left(\begin{array}{l}
r \\
t
\end{array}\right)\left(\gamma_{\mathrm{th}}+1\right)^{r-t} \\
& \times \int_{0}^{\infty} \bar{z}^{s+t-r} \mathrm{e}^{-c_{1}\left(\gamma_{\mathrm{th}}+1\right) \bar{z}^{-1}-j_{1} \bar{z} e_{\mathrm{d}, 1}^{-1}} d \bar{z}
\end{aligned}
$$

where the integral can be evaluated with the help of (54). Thus, after substituting (59) and (58) into (57), we obtain (20).

\section{APPENDIX E}

\section{DERIVATION OF (29)}

For notational simplicity, we define $\hat{\alpha}_{1} \triangleq e_{\mathrm{d}, 1} \alpha_{\mathrm{d}, 1}, \hat{\alpha}_{2} \triangleq$ $g_{\mathrm{s}, 1} \alpha_{\mathrm{s}, 1}$ and $\hat{\alpha}_{0} \triangleq g_{\mathrm{s}, 2} \alpha_{\mathrm{s}, 2}$. The first part of $I_{\mathrm{e}}$ in (28) can be expressed in terms of double integration as

$$
I_{\mathrm{e}, 1}=\int_{0}^{y_{\mathrm{u}}} \int_{0}^{z_{\mathrm{u}}} \operatorname{Pr}\left\{\hat{\alpha}_{2} \geq y, \hat{\alpha}_{2} \geq z, z \leq \gamma_{\mathrm{th}}\right\} f_{\hat{\alpha}_{1}}(z) d z f_{\hat{\alpha}_{0}}(y) d y
$$

where $y_{\mathrm{u}}$ and $z_{\mathrm{u}}$ are the upper limits for $y$ and $z$, respectively. It is obvious from (60) that $\operatorname{Pr}\left\{\hat{\alpha}_{2} \geq y, \hat{\alpha}_{2} \geq z, z \leq \gamma_{\text {th }}\right\}$ reduces to $\operatorname{Pr}\left\{\hat{\alpha}_{2} \geq y, \hat{\alpha}_{2} \geq z\right\}$ when the condition $z \leq \gamma_{\text {th }}$ is fulfilled, for which $z_{\mathrm{u}}=\gamma_{\mathrm{th}}$. However, $y_{\mathrm{u}}$ can go to infinity. The resulting integral in (60) can be separated into two parts, for $0 \leq y \leq \gamma_{\mathrm{th}}$ and $\gamma_{\mathrm{th}} \leq y \leq \infty$. For $0 \leq y \leq \gamma_{\mathrm{th}}$, (60) can be expressed as

$$
\left[I_{\mathrm{e}, 1}\right]_{y=0}^{\gamma_{\mathrm{th}}}=\int_{y=0}^{\gamma_{\mathrm{th}}} \int_{z=0}^{\gamma_{\mathrm{th}}} \operatorname{Pr}\left\{\hat{\alpha}_{2} \geq y, \hat{\alpha}_{2} \geq z\right\} f_{\hat{\alpha}_{1}}(z) d z f_{\hat{\alpha}_{0}}(y) d y .
$$

We know that $\operatorname{Pr}\left\{\hat{\alpha}_{2} \geq y, \hat{\alpha}_{2} \geq z\right\}$ in (61) can take the following values:

$$
\operatorname{Pr}\left\{\hat{\alpha}_{2} \geq y, \hat{\alpha}_{2} \geq z\right\}= \begin{cases}\operatorname{Pr}\left\{\hat{\alpha}_{2} \geq z\right\} & \text { for } z \geq y \\ \operatorname{Pr}\left\{\hat{\alpha}_{2} \geq y\right\} & \text { for } z \leq y\end{cases}
$$

With the help of (62), (61) can be expressed as

$$
\begin{aligned}
{\left[I_{\mathrm{e}, 1}\right]_{y=0}^{\gamma_{\mathrm{th}}}=} & \int_{y=0}^{\gamma_{\mathrm{th}}} \int_{z=y}^{\gamma_{\mathrm{th}}} \operatorname{Pr}\left\{\hat{\alpha}_{2} \geq z\right\} f_{\hat{\alpha}_{1}}(z) d z f_{\hat{\alpha}_{0}}(y) d y \\
& +\int_{z=0}^{\gamma_{\mathrm{th}}} \int_{y=z}^{\gamma_{\mathrm{th}}} \operatorname{Pr}\left\{\hat{\alpha}_{2} \geq y\right\} f_{\hat{\alpha}_{1}}(z) d z f_{\hat{\alpha}_{0}}(y) d y
\end{aligned}
$$

which after simple steps can be rewritten as

$$
\begin{aligned}
{\left[I_{\mathrm{e}, 1}\right]_{y=0}^{\gamma_{\mathrm{th}}}=} & 2 \operatorname{Pr}\left\{\hat{\alpha}_{1} \leq \gamma_{\mathrm{th}}\right\} \operatorname{Pr}\left\{\hat{\alpha}_{0} \leq \gamma_{\mathrm{th}}\right\} \\
& -\int_{0}^{\gamma_{\mathrm{th}}} \operatorname{Pr}\left\{\hat{\alpha}_{1}\right\} f_{\hat{\alpha}_{0}}(y) d y-\int_{0}^{\gamma_{\mathrm{th}}} \operatorname{Pr}\left\{\hat{\alpha}_{0} \leq z\right\} f_{\hat{\alpha}_{1}}(z) d z \\
& -\int_{y=0}^{\gamma_{\mathrm{th}}} \int_{z=y}^{\gamma_{\mathrm{th}}} \operatorname{Pr}\left\{\hat{\alpha}_{2} \leq z\right\} f_{\hat{\alpha}_{1}}(z) d z f_{\hat{\alpha}_{0}}(y) d y \\
& -\int_{z=0}^{\gamma_{\mathrm{th}}} \int_{y=z}^{\gamma_{\mathrm{th}}} \operatorname{Pr}\left\{\hat{\alpha}_{2} \leq y\right\} f_{\hat{\alpha}_{1}}(z) d z f_{\hat{\alpha}_{0}}(y) d y .
\end{aligned}
$$

Applying (25) into (64), and after some lengthy but straightforward derivations, (64) can be expressed as

$$
\begin{aligned}
{\left[I_{\mathrm{e}, 1}\right]_{y=0}^{\gamma_{\mathrm{th}}}=} & \frac{r_{\mathrm{s}, 2} r_{\mathrm{d}, 1} \gamma_{\mathrm{th}}^{\mu_{\mathrm{s}, 2}}+\mu_{\mathrm{d}, 1}}{e_{\mathrm{d}, 1}^{\mu_{\mathrm{d}, 1}} g_{\mathrm{s}, 2}^{\mu_{\mathrm{s}}} \mu_{\mathrm{s}, 2} \mu_{\mathrm{d}, 1}} \\
& -\frac{r_{\mathrm{s}, 2} r_{\mathrm{d}, 1} r_{\mathrm{s}, 1}}{e_{\mathrm{d}, 1} g_{\mathrm{s}, 2}^{\mu_{\mathrm{s}, 2}}} \frac{\gamma_{\mathrm{th}}^{\mu_{\mathrm{s}, 2}+\mu_{\mathrm{d}, 1}+\mu_{\mathrm{s}, 1}}}{g_{\mathrm{s}, 1}^{\mu_{\mathrm{s}, 1}} \mu_{\mathrm{s}, 1}\left(\mu_{\mathrm{s}, 2}+\mu_{\mathrm{d}, 1}+\mu_{\mathrm{s}, 1}\right)} \\
& \times\left[\frac{1}{\mu_{\mathrm{s}, 2}}+\frac{1}{\mu_{\mathrm{d}, 1}}\right] .
\end{aligned}
$$

Since $z_{\mathrm{u}}=\gamma_{\mathrm{th}}$, for $\gamma_{\mathrm{th}} \leq y \leq \infty, \operatorname{Pr}\left\{\hat{\alpha}_{2} \geq y, \hat{\alpha}_{2} \geq z\right\}=$ $\operatorname{Pr}\left\{\hat{\alpha}_{2} \geq y\right\}$, and thus (60) can be expressed as

$$
\left[I_{\mathrm{e}, 1}\right]_{y=\gamma_{\mathrm{th}}}^{\infty}=\int_{y=\gamma_{\mathrm{th}}}^{\infty} \int_{z=0}^{\gamma_{\mathrm{th}}} \operatorname{Pr}\left\{\hat{\alpha}_{2} \geq y\right\} f_{\hat{\alpha}_{1}}(z) d z f_{\hat{\alpha}_{0}}(y) d y
$$

which can be re-expressed as

$$
\begin{aligned}
{\left[I_{\mathrm{e}, 1}\right]_{y=\gamma_{\mathrm{th}}}^{\infty}=- } & \operatorname{Pr}\left\{\hat{\alpha}_{0} \leq \gamma_{\mathrm{th}}\right\} \operatorname{Pr}\left\{\hat{\alpha}_{1} \leq \gamma_{\mathrm{th}}\right\}+\operatorname{Pr}\left\{\hat{\alpha}_{1} \leq \gamma_{\mathrm{th}}\right\} \\
& \times\left(1-\int_{y=\gamma_{\mathrm{th}}}^{\infty} \operatorname{Pr}\left\{\hat{\alpha}_{2} \leq y\right\} f_{\hat{\alpha}_{0}}(y) d y\right)
\end{aligned}
$$

Using the following relations:

$$
\begin{array}{r}
\int_{y=0}^{\gamma_{\text {th }}} \operatorname{Pr}\left\{\hat{\alpha}_{2} \leq y\right\} f_{\hat{\alpha}_{0}}(y) d y+\int_{y=\gamma_{\text {th }}}^{\infty} \operatorname{Pr}\left\{\hat{\alpha}_{2} \leq y\right\} f_{\hat{\alpha}_{0}}(y) d y \\
=\operatorname{Pr}\left\{\hat{\alpha}_{2} \leq \hat{\alpha}_{0}\right\}=1-\operatorname{Pr}\left\{\hat{\alpha}_{2} \geq \hat{\alpha}_{0}\right\},
\end{array}
$$

(68) can be rewritten as

$$
\begin{aligned}
{\left[I_{\mathrm{e}, 1}\right]_{y=\gamma_{\mathrm{th}}}^{\infty}=} & -\operatorname{Pr}\left\{\hat{\alpha}_{0} \leq \gamma_{\mathrm{th}}\right\} \operatorname{Pr}\left\{\hat{\alpha}_{1} \leq \gamma_{\mathrm{th}}\right\}+\operatorname{Pr}\left\{\hat{\alpha}_{1} \leq \gamma_{\mathrm{th}}\right\} \\
& \times \operatorname{Pr}\left\{\hat{\alpha}_{2} \geq \hat{\alpha}_{0}\right\}+\operatorname{Pr}\left\{\hat{\alpha}_{1} \leq \gamma_{\mathrm{th}}\right\} \\
& \times \int_{y=0}^{\gamma_{\mathrm{th}}} \operatorname{Pr}\left\{\hat{\alpha}_{2} \leq y\right\} f_{\hat{\alpha}_{0}}(y) d y .
\end{aligned}
$$

Substituting (25) into (69) and after some steps, we obtain

$$
\begin{aligned}
{\left[I_{\mathrm{e}, 1}\right]_{y=\gamma_{\mathrm{th}}}^{\infty}=} & -\frac{r_{\mathrm{s}, 2} r_{\mathrm{d}, 1} \gamma_{\mathrm{th}}^{\mu_{\mathrm{s}, 2}+\mu_{\mathrm{d}, 1}}}{\mu_{\mathrm{s}, 2} \mu_{\mathrm{d}, 1} g_{\mathrm{s}, 2}^{\mu_{\mathrm{s}, 2}} e_{\mathrm{d}, 1}^{\mu_{\mathrm{d}, 1}}}+\frac{r_{\mathrm{d}, 1} \gamma_{\mathrm{th}}^{\mu_{\mathrm{d}, 1}}}{\mu_{\mathrm{d}, 1} e_{\mathrm{d}, 1}^{\mu_{\mathrm{d}}}} \\
& \times \operatorname{Pr}\left\{\hat{\alpha}_{2} \geq \hat{\alpha}_{0}\right\}+\frac{r_{\mathrm{s}, 2} r_{\mathrm{d}, 1} r_{\mathrm{s}, 1}}{\mu_{\mathrm{d}, 1} \mu_{\mathrm{s}, 1}\left(\mu_{\mathrm{s}, 2}+\mu_{\mathrm{s}, 1}\right)} \\
& \times \frac{\gamma_{\mathrm{th}, 2}^{\mu_{\mathrm{s}, 2}+\mu_{\mathrm{d}, 1}+\mu_{\mathrm{s}, 1}}}{g_{\mathrm{s}, 2}^{\mu_{\mathrm{s}, 2}} e_{\mathrm{d}, 1}^{\mu_{\mathrm{d}, 1}} g_{\mathrm{s}, 1}^{\mu_{\mathrm{s}, 1}}} .
\end{aligned}
$$

Summing (65) and (70), we obtain (29).

\section{APPENDIX F}

DERIVATION OF (30)

The second part of $I_{\mathrm{e}}$ in (28) can be expressed in terms of double integration as

$$
I_{\mathrm{e}, 2}=\int_{0}^{y_{\mathrm{u}}} \int_{0}^{z_{\mathrm{u}}} \operatorname{Pr}\left\{\hat{\alpha}_{2} \leq \gamma_{\mathrm{th}}, \hat{\alpha}_{2} \geq y, \hat{\alpha}_{2} \leq z\right\} f_{\hat{\alpha}_{1}}(z) d z f_{\hat{\alpha}_{0}}(y) d y .
$$


In (71), it is clear that $z_{11}=\infty$. We rewrite the integral of (71) as the sum of two integrals where $0 \leq z \leq \gamma_{\mathrm{th}}$ and $\gamma_{\mathrm{th}} \leq z \leq \infty$. Thus, we have

$$
\begin{aligned}
I_{\mathrm{e}, 2}=\int_{y=0}^{y_{\mathrm{u}}} \int_{z=0}^{\gamma_{\mathrm{th}}} \operatorname{Pr}\left\{\hat{\alpha}_{2} \leq \gamma_{\mathrm{th}}, \hat{\alpha}_{2} \geq y, \hat{\alpha}_{2} \leq z\right\} \\
\quad \times f_{\hat{\alpha}_{1}}(z) d z f_{\hat{\alpha}_{0}}(y) d y \\
+\int_{y=0}^{y_{\mathrm{u}}} \int_{z=\gamma_{\mathrm{th}}}^{\infty} \operatorname{Pr}\left\{\hat{\alpha}_{2} \leq \gamma_{\mathrm{th}}, \hat{\alpha}_{2} \geq y, \hat{\alpha}_{2} \leq z\right\} \\
\times f_{\hat{\alpha}_{1}}(z) d z f_{\hat{\alpha}_{0}}(y) d y .
\end{aligned}
$$

It is not difficult to show that $\tilde{P}_{\mathrm{A}} \triangleq \operatorname{Pr}\left\{\hat{\alpha}_{2} \leq \gamma_{\mathrm{th}}, \hat{\alpha}_{2} \geq y, \hat{\alpha}_{2} \leq\right.$ $z$ \} can be expressed as

$$
\tilde{P}_{\mathrm{A}}= \begin{cases}\operatorname{Pr}\left\{\hat{\alpha}_{2} \geq y, \hat{\alpha}_{2} \leq z\right\} & \text { for } z \leq \gamma_{\mathrm{th}} \\ \operatorname{Pr}\left\{\hat{\alpha}_{2} \geq y, \hat{\alpha}_{2} \leq \gamma_{\mathrm{th}}\right\} & \text { for } z \geq \gamma_{\mathrm{th}}\end{cases}
$$

Also, note that $\operatorname{Pr}\left\{\hat{\alpha}_{2} \leq z, \hat{\alpha}_{2} \geq y\right\}$ is nonzero for $y \leq z$. Hence, using (73), (72) can be rewritten as

$$
\begin{aligned}
I_{\mathrm{e}, 2}= & \int_{z=0}^{\gamma_{\mathrm{th}}} \int_{y=0}^{z}\left(\operatorname{Pr}\left\{\hat{\alpha}_{2} \leq z\right\}-\operatorname{Pr}\left\{\hat{\alpha}_{2} \leq y\right\}\right) f_{\hat{\alpha}_{1}}(z) d z f_{\hat{\alpha}_{0}}(y) d y \\
& +\int_{y=0}^{\gamma_{\mathrm{th}}} \int_{z=\gamma_{\mathrm{th}}}^{\infty} \operatorname{Pr}\left\{\hat{\alpha}_{2} \leq \gamma_{\mathrm{th}}, \hat{\alpha}_{2} \geq y\right\} f_{\hat{\alpha}_{1}}(z) d z f_{\hat{\alpha}_{0}}(y) d y .
\end{aligned}
$$

Now, with the help of (25) and after some derivations, the first and second integrals of (74) can be expressed as

$$
\begin{aligned}
{\left[I_{\mathrm{e}, 2}\right]_{z=0}^{\gamma_{\mathrm{th}}}=} & \frac{r_{\mathrm{s}, 2} r_{\mathrm{d}, 1} r_{\mathrm{s}, 1}}{\mu_{\mathrm{s}, 2}\left(\mu_{\mathrm{s}, 2}+\mu_{\mathrm{s}, 1}\right)} \\
& \times \frac{\gamma_{\mathrm{sh}, 2}^{\mu_{\mathrm{s}, 1}+\mu_{\mathrm{s}, 1}}}{\left(\mu_{\mathrm{s}, 2}+\mu_{\mathrm{d}, 1}+\mu_{\mathrm{s}, 1}\right) g_{\mathrm{s}, 2}^{\mu_{\mathrm{s}, 2}} e_{\mathrm{d}, 1}^{\mu_{\mathrm{d}, 1}} g_{\mathrm{s}, 1}^{\mu_{\mathrm{s}, 1}}} \\
{\left[I_{\mathrm{e}, 2}\right]_{z=\gamma_{\mathrm{th}}}^{\infty}=} & \frac{r_{\mathrm{s}, 2} r_{\mathrm{s}, 1} \gamma_{\mathrm{th}}^{\mu_{\mathrm{s}, 2}+\mu_{\mathrm{s}, 1}}}{\mu_{\mathrm{s}, 2}\left(\mu_{\mathrm{s}, 2}+\mu_{\mathrm{s}, 1}\right) g_{\mathrm{s}, 2}^{\mu_{\mathrm{s}, 2}} g_{\mathrm{s}, 1}^{\mu_{\mathrm{s}, 1}}}-\frac{r_{\mathrm{s}, 2} r_{\mathrm{d}, 1}}{\mu_{\mathrm{d}, 1}\left(\mu_{\mathrm{s}, 2}+\mu_{\mathrm{s}, 1}\right)} \\
& \times \frac{r_{\mathrm{s}, 1} \gamma_{\mathrm{th}}^{\mu_{\mathrm{s}, 2}+\mu_{\mathrm{d}, 1}+\mu_{\mathrm{s}, 1}}}{\mu_{\mathrm{s}, 2} g_{\mathrm{s}, 2}^{\mu_{\mathrm{s}, 2}} e_{\mathrm{d}, 1}^{\mu_{\mathrm{d}, 1}} g_{\mathrm{s}, 1}^{\mu_{\mathrm{s}, 1}}}
\end{aligned}
$$

After adding (75) and (76), we get the expression (30).

\section{REFERENCES}

[1] A. S. Avestimehr and D. N. C. Tse, "Outage capacity of the fading relay channel in the low SNR regime," IEEE Trans. Inf. Theory, vol. 53, no. 4, pp. 1401-1415, Apr. 2007.

[2] J. N. Laneman and G. W. Wornell, "Exploiting distributed spatial diversity in wireless networks," presented at the Allerton Conf. Commun., Control, Comput., Monticello, IL, Oct. 2000.

[3] M. Janani, A. Hedayat, T. E. Hunter, and A. Nosratinia, "Coded cooperation in wireless communications: Space-time transmission and iterative decoding," IEEE Trans. Signal Process., vol. 52, pp. 362-371, Feb. 2004.

[4] G. Kramer, M. Gastpar, and P. Gupta, "Cooperative strategies and capacity theorem for relay networks," IEEE Trans. Inf. Theory, vol. 51, pp. 3037-3063, Sep. 2005.

[5] A. Blestas, A. Khisti, D. P. Reed, and A. Lippman, "A simple cooperative diversity method based on network path selection," IEEE J. Sel. Areas Commun., vol. 24, no. 3, pp. 659-672, Mar. 2006.

[6] A. Blestas, H. Shin, and M. Z. Win, "Cooperative communications with outage optimal opportuinistic relaying," IEEE Trans. Wireless Commun., vol. 6, pp. 3450-3460, Sep. 2007.
[7] Y. Zhao, R. Adve, and T. J. Lim, "Symbol error rate of selection amplify-and-forward relay systems," IEEE Commun. Lett., vol. 10, no. 11, pp. 757-759, Nov. 2006.

[8] A. S. Ibrahim, A. K. Sadek, W. Su, and K. J. R. Liu, "Cooperative communications with relay-selection: When to cooperate and whom to cooperate with?," IEEE Trans. Wireless Commun., vol. 7, no. 7, pp. 2814-2827, Jul. 2008.

[9] Y. Zhao, R. Adve, and T. J. Lim, "Improving amplify-and-forward relay networks: Optimal power allocation versus selection," IEEE Trans. Wireless Commun., vol. 6, no. 8, pp. 3114-3123, Aug. 2007.

[10] Y. Jing and H. Jafarkhani, "Single and multiple relay selection schemes and their achievable diversity order," IEEE Trans. Wireless Commun., vol. 7, pp. 1414-1423, Mar. 2009.

[11] B. Wang, J. Zhang, and A. Host-Madsen, "On the capacity of MIMO relay channels," IEEE Trans. Inf. Theory, vol. 51, pp. 29-43, Jan. 2005.

[12] M. Yuksel and E. Erkip, "Multiple-antenna cooperative wireless systems: A diversity-multiplexing tradeoff perspective," IEEE Trans. Inf. Theory, vol. 53, pp. 3371-3393, Oct. 2007.

[13] I.-H. Lee and D. Kim, "Outage probability of multi-hop MIMO relaying with transmit antenna selection and ideal relay gain over Rayleigh fading channels," IEEE Trans. Commun., vol. 57, pp. 357-360, Feb. 2009.

[14] M. Ju, H.-K. Song, and I.-M. Kim, "Joint relay-and-antenna selection in multi-antenna relay networks," IEEE Trans. Commun., vol. 58, no. 12, pp. 3417-3422, Dec. 2010.

[15] K.-J. Lee, H. Sung, E. Park, and I. Lee, "Joint optimization for one and two-way MIMO AF multiple-relay systems," IEEE Trans. Wireless Commun., vol. 9, no. 12, pp. 3671-3681, Dec. 2010

[16] M. A. Torabi and J.-F. Frigon, "Semi-orthogonal relay selection and beamforming for amplify-and-forward MIMO relay channels," in Proc. IEEE WCNC, 2008, pp. 48-53.

[17] Z. Han and K. J. R. Liu, Resource Allocation for Wireless Networks: Basics, Techniques and Applications. Cambridge, U.K.: Cambridge Univ. Press, 2008.

[18] I. Krikidis, J. Thompson, S. McLaughlin, and N. Goertz, "Amplifyand-forward with partial relay selection," IEEE Commun. Lett., vol. 12, no. 4, pp. 235-237, Apr. 2008.

[19] J.-B. Kim and D. Kim, "Comparison of tightly power-constrained performances for opportunistic amplify-and-forward relaying with partial or full channel information," IEEE Commun. Lett., vol. 13, no. 2, pp. 100-102, Feb. 2009

[20] D. B. da Costa and S. Aissa, "End-to-end performance of dual-hop semi-blind relaying systems with partial relay selection," IEEE Trans. Wireless Commun., vol. 8, no. 8, pp. 4306-4315, Aug. 2009.

[21] D. B. da Costa and S. Aissa, "Capacity analysis of cooperative systems with relay selection in Nakagami-m fading," IEEE Commun. Lett., vol. 13, no. 9, pp. 637-639, Sep. 2009.

[22] L. Sun, T. Zhang, H. Niu, and J. Wang, "Effect of multiple antennas at the destination on the diversity performance of amplify-and-forward systems with partial relay selection," IEEE Signal Process. Lett., vol. 17, no. 7, pp. 631-634, Jul. 2010.

[23] B. Khoshnevis, W. Yu, and R. Adve, "Grassmannian beamforming for MIMO amplify-and-forward relaying," IEEE J. Sel. Areas Commun., vol. 26, no. 8, pp. 1397-1407, Oct. 2008.

[24] B. Hassibi and B. M. Hochwald, "How much training is needed in multiple-antenna wireless links?," IEEE Trans. Inf. Theory, vol. 49, no. 4, pp. 951-963, Apr. 2003.

[25] P. Uthansakul, N. Promsuwanna, and M. Uthansakul, "Performance of antenna selection in MIMO system using channel reciprocity with measured data," Int. J. Antennas Propag., vol. 2011, Article ID 854350

[26] J. Joung and A. H. Sayed, "Design of half- and full-duplex relay systems based on the MMSE formulation," in Proc. IEEE/SP 15th Workshop Stat. Signal Process., 2009, pp. 281-284.

[27] T. Riihonen, S. Werner, and R. Wichman, "Spatial loop interference suppression in full-duplex MIMO relays," presented at the Annu. Asilomar Conf. Signals, Syst., Comput., Pacific Grove, CA, Nov. 2009.

[28] C. Xing, S. Ma, and Y.-C. Wu, "Robust joint design of linear precoder and destination equalizer for dual-hop amplify-and-forward MIMO relay systems," IEEE Trans. Signal Process., vol. 58, no. 4, pp. 2273-2283, Apr. 2010.

[29] P. A. Dighe, R. K. Mallik, and S. S. Jamuar, "Analysis of transmitreceive diversity in Rayleigh fading," IEEE Trans. Commun., vol. 15, no. 4, pp. 694-703, Apr. 2003.

[30] I. S. Gradshteyn and I. M. Ryzhik, Table of Integrals, Series, and Products. New York: Academic, 2000

[31] M. O. Hasna and M.-S. Alouini, "End-to-end performance of transmission systems with relays over Rayleigh-fading channels," IEEE Trans. Wireless Commun., vol. 2, no. 6, pp. 1126-1131, Nov. 2003.

[32] Q. Liu, X. Ma, and G. T. Zhou, "General diversity gain function and its application in amplify-and-forward cooperative networks," IEEE Trans. Signal Process., vol. 59, no. 2, pp. 859-863, Feb. 2011.

[33] Q. Zhou and H. Dai, "Asymptotic analysis in MIMO MRT/MRC systems," EURASIP J. Wireless Commun. Netw., vol. 2006, pp. 1-8, Article ID 45831. 
[34] Y. Liang, "MIMO relay wireless networks with scheduling and transmit beamforming," Wireless Commun. and Mob. Comput., vol. 12, no. 4, pp. 345-351, Mar. 2012.

[35] P. A. Anghel and M. Kaveh, "Exact symbol error probability of a cooperative network in a Rayleigh-fading environment," IEEE Trans. Wireless Commun., vol. 3, no. 5, pp. 1416-1421, Sep. 2004.

[36] A. Papoulis, Probability, Random Variables, and Stochastic Processes. New York: McGraw-Hill, 1991.

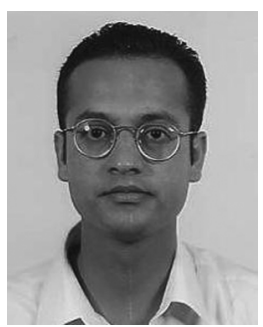

Batu Krishna Chalise (SM'11) was born in Kathmandu, Nepal. He received the B.E. degree in electronics engineering from Tribhuvan University, Kathmandu, in 1998 and the M.S. and Ph.D. degrees in electrical engineering from the University of Duisburg-Essen, Duisburg, Germany, in 2001 and 2006, respectively.

In 1999, he was a Lecturer with the Institute of Engineering, Kathmandu. For a short period in 2001, he was a Researcher with the Fraunhofer-Institute of Microelectronic Circuits and Systems (IMS), Duisburg. From 2002 to 2006, he was a Research Assistant with the Department of Communication Systems, University of Duisburg-Essen, where his Ph.D. research was supported by a grant from the Ministry of Education and Science of North Rhein-Westphalia (NRW), Germany. He was a Postdoctoral Researcher with the Communication and Remote Sensing Laboratory, Universitè Catholique de Louvain, Louvain La Neuve, Belgium, from December 2006 until June 2010 Currently, he is the Postdoctoral Research Fellow with the Center for Advanced Communications, Villanova University, Villanova, PA. His research interests include cooperative and opportunistic wireless communications, robust algorithms for multiantenna systems, and convex optimization.

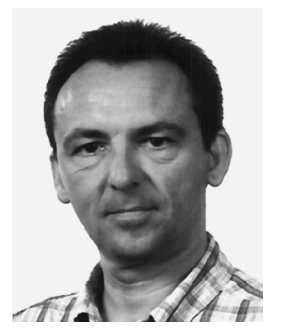

Luc Vandendorpe (F'06) was born in Mouscron, Belgium, in 1962. He received the Electrical Engineering degree (summa cum laude) and the $\mathrm{Ph} . \mathrm{D}$. degree from the Universit Catholique de Louvain (UCL) Louvain-la-Neuve, Belgium, in 1985 and 1991, respectively.

Since 1985, he has been with the Communications and Remote Sensing Laboratory of UCL, where he first worked in the field of bit rate reduction techniques for video coding. In 1992, he was a Visiting Scientist and Research Fellow at the Telecommunications and Traffic Control Systems Group of the Delft Technical University, Delft, The Netherlands, where he worked on spread spectrum techniques for personal communications systems. From October 1992 to August 1997, he was Senior Research Associate of the Belgian NSF at UCL. Presently, he is Full Professor and Head of the Institute for Information and Communication Technologies, Electronics and Applied Mathematics of UCL. His current interest is in digital communication systems and more precisely resource allocation for OFDM(A) based multicell systems, MIMO and distributed MIMO, sensor networks, turbo-based communications systems, physical layer security, and UWB based positioning.

Dr. Vandendorpe was corecipient of the Biennal Alcatel-Bell Award from the Belgian NSF for a contribution in the field of image coding in 1990. In 2000, he was corecipient (with J. Louveaux and F. Deryck) of the Biennal Siemens Award from the Belgian NSF for a contribution about filter bank based multicarrier transmission. In 2004, he was co-winner (with J. Czyz) of the Face Authentication Competition, FAC 2004. He is or has been TPC member for numerous IEEE conferences (VTC Fall, Globecom Communications Theory Symposium, SPAWC, and ICC) and for the Turbo Symposium. He was Co-Technical Chair (with P. Duhamel) for the IEEE ICASSP 2006 He was an Editor of the IEEE TRANSACTIONS ON COMMUNICATIONS FOR SYNCHRONIZATION AND EQUALIZATION between 2000 and 2002, Associate Editor of the IEEE TRANSACTIONS ON WIRELESS COMMUNICATIONS between 2003 and 2005, and Associate Editor of the IEEE TRANSACTIONS ON SignAL PROCESSING between 2004 and 2006. He was Chair of the IEEE Benelux joint chapter on Communications and Vehicular Technology between 1999 and 2003. He was an elected member of the Signal Processing for Communications Committee between 2000 and 2005, and between 2009 and 2011, and an elected member of the Sensor Array and Multichannel Signal Processing Committee of the Signal Processing Society between 2006 and 2008. Currently, he is the Editor-in-Chief for the EURASIP Journal on Wireless Communications and Networking.

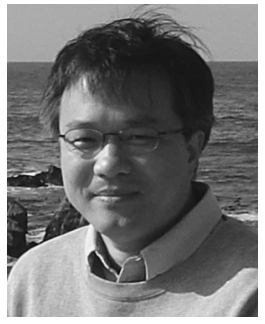

Yimin D. Zhang (SM'01) received the Ph.D. degree from the University of Tsukuba, Tsukuba, Japan, in 1988.

He joined the faculty of the Department of Radio Engineering, Southeast University, Nanjing, China, in 1988. He served as a Technical Manager at the Communication Laboratory Japan, Kawasaki, Japan, from 1995 to 1997, and was a Visiting Researcher at ATR Adaptive Communications Research Laboratories, Kyoto, Japan, from 1997 to 1998. Since 1998 he has been with the Villanova University, Villanova, PA, where he is currently a Research Professor with the Center for Advanced Communications and the Director of the Wireless Communications and Positioning Laboratory. He has more than 170 publications in the area of statistical signal and array processing for communications and radar applications, including digital mobile communications, wireless networks, over-the-horizon radar, MIMO systems, cooperative communications, blind signal processing, jammer suppression, time-frequency analysis, source localization and target tracking, radio frequency identification (RFID), and image processing.

Dr. Zhang is an Associate Editor for the Journal of the Franklin Institute and serves on the Editorial Board of the Signal Processing journal. He is an Associate Editor for the IEEE TRANSACTIONS ON SIGNAL PROCESSING and was an Associate Editor for the IEEE Signal Processing LetTERs from 2006 to 2010 .

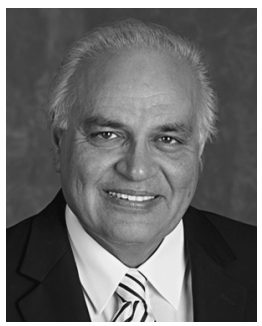

Moeness G. Amin (F'01) received the Ph.D. degree in Electrical Engineering from the University of Colorado, Boulder, in 1984.

He has been on the Faculty of the Department of Electrical and Computer Engineering at Villanova University, Villanova, PA, since 1985. In 2002, he became the Director of the Center for Advanced Communications, College of Engineering. He has more than 450 journal and conference publications in the areas of wireless communications, time-frequency analysis, smart antennas, waveform design and diversity, interference cancellation in broadband communication platforms, anti-jam GPS, target localization and tracking, direction finding, channel diversity and equalization, ultrasound imaging, and radar signal processing.

Dr. Amin is the Recipient of the 2009 Individual Technical Achievement Award from the European Association of Signal Processing, and the Recipient of the 2010 NATO Scientific Achievement Award. He is a Fellow of the International Society of Optical Engineering, 2007, and a Fellow of the Institute of Engineering and Technology (IET), 2010. He is a recipient of the IEEE Third Millennium Medal, 2000; recipient of the Chief of Naval Research Challenge Award, 2010; Distinguished Lecturer of the IEEE Signal Processing Society, 2003-2004; Active Member of the Franklin Institute Committee on Science and the Arts; recipient of the Villanova University Outstanding Faculty Research Award, 1997; and the recipient of the IEEE Philadelphia Section Award, 1997. He is a member of SPIE, EURASIP, ION, Eta Kappa Nu, Sigma Xi, and Phi Kappa Phi. He currently serves on the Overview Board of the IEEE TRANSACTIONS ON SignAl PROCESSING as well as on the Editorial Board of the EURASIP Signal Processing Journal. He was a Plenary Speaker at ICASSP 2010. He was the Special Session Co-Chair of the 2008 IEEE International Conference on Acoustics, Speech, and Signal Processing. He was the Technical Program Chair of the Second IEEE International Symposium on Signal Processing and Information Technology, 2002. He was the General and Organization Chair of the IEEE Workshop on Statistical Signal and Array Processing, 2000. He was the General and Organization Chair of the IEEE International Symposium on Time-Frequency and Time-Scale Analysis, 1994. He was an Associate Editor of the IEEE TRANSACTIONS ON SigNAL PROCESSING during 1996-1998. He was a member of the IEEE Signal Processing Society Technical Committee on Signal Processing for Communications during 1998-2002. He was a Member of the IEEE Signal Processing Society Technical Committee on Statistical Signal and Array Processing during 1995-1997. He has given several keynote and plenary talks and served as a Session Chair in several technical meetings. He was the Guest Editor of the Journal of the Franklin Institute Special Issue on Advances in Indoor Radar Imaging in September 2008. He was a Guest Editor of the IEEE TRAnsactions on GEOSCIENCE AND Remote SEnSING Special Issue on Remote Sensing of Building Interior in May 2009, and a Guest Editor of the ET Signal Processing Special Issue on Time-Frequency Approach to Radar Detection, Imaging, and Classification in December 2009. 